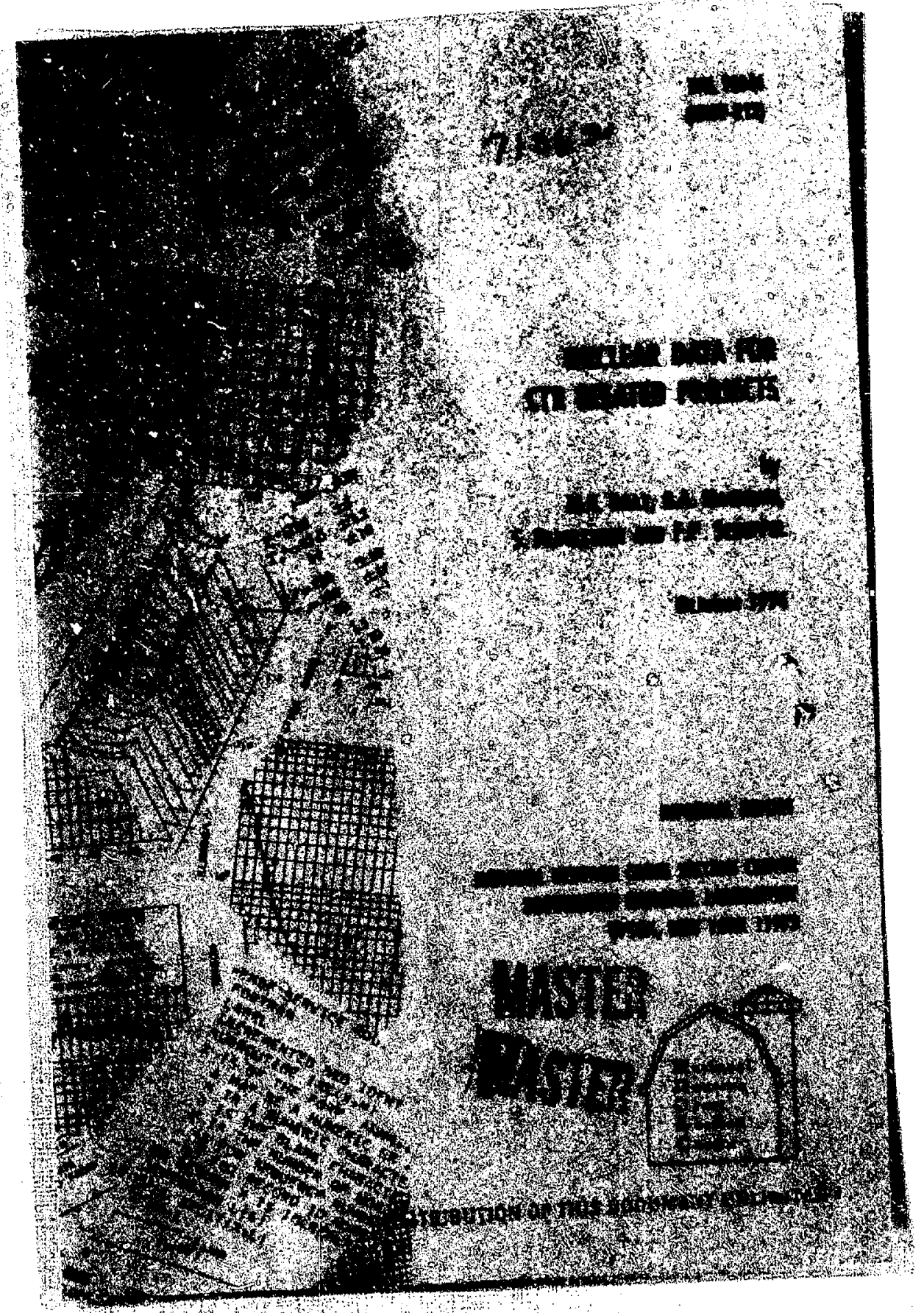




\title{
NUCLEAR DATA FOR CTR RELATED PROJECTS
}

\author{
by
}

\author{
M.R. Bhat, B.A. maguino, S. Pearlstein aho F.M. Scheffel
}

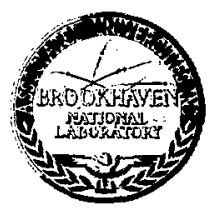

\section{October 1974}

\section{NATIONAL NEUTRON CROSS SECTION CENTER \\ BROOKHAVEN NATIONAL LABORATORY ASSOCIATED UNIVERSITIES, INC. UNDER CONTRACT NO. AT/30-1 - 16 WIH THE \\ UNITED STATES ATOMIC ENERGY COMWISSION} Thaneored by the Unitod States Govor Atomic Enetcy the Unked Stetes nor the Uniced States A nor any of Comalision, nor any of their employes, employees, theit contractors, subcontract ors, of ind or aseumes eny tmkes any wartanty, express or implied, or ascury, comlegl linbulty or responsibility for the eccuracy, cometus, plateness or usofulness of any toreptesents that its ase product or proces. diclosed, or sed rights. 


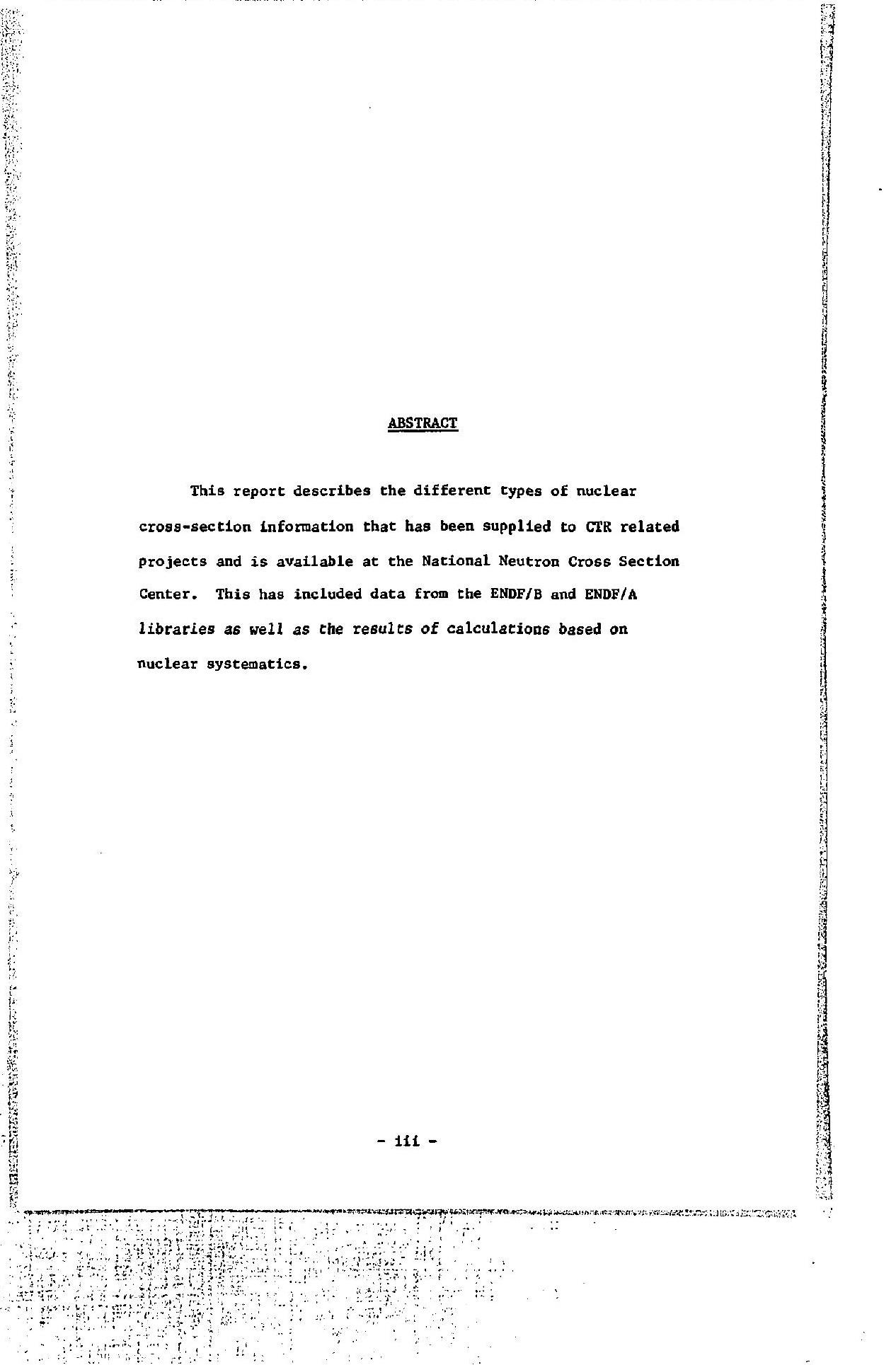




\section{Inthar cantate}

past

1. Incroduction .......................... 1

2. Muctear Crost-5ectione tor the che Program .......

3. Llst of cth hequates ................. 10

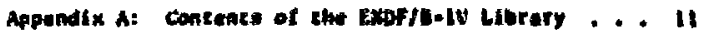

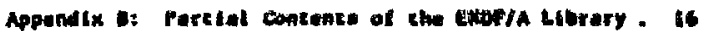

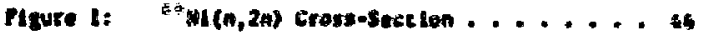

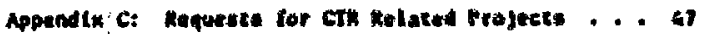

Aetarencus .................. ss

$-1 v=$ 


\section{Introduces lon}

The Mational Neutron Crase Section Canter (Mnusc) hos provided ctons-action daca for a large number of nuclet of interest to the

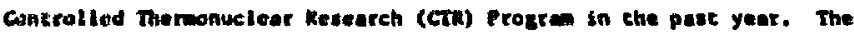
data wre upplied to a small nuber of laborncorles actively engaged In the CTh progran and had a limtend distribution. This report sumarizes this volemtmous body of material by gtutng (a) soutces of partisl or

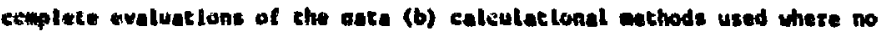
etroluation or experimental date axtse (c) list of melet for which the

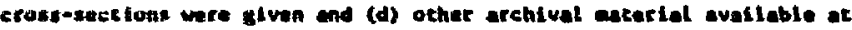

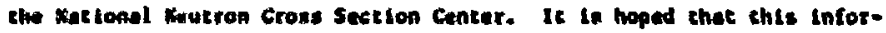
wation would be of faterest to a largex elses of usars and indicate the type of date avallable from tho kicsc. 


\section{Nuclenr Crose-Sections for the CTR Program}

There is at present a large amount of research activity connected with the CTR program at can be seen from the proceedings of a recent conferercé: Sowe of the areae of Interest in this program are (1) tritium breeding (2) energy deposition (3) anterial activation (4) helfum and hydrogen production (5) atomic displacement and materials damage

(6) dosimetry and eransmutation analyals (7) fission-fusion systems and

(8) fistion product burner systens. These studies need nuclear data of different types, degree of detail and accuracy. In some cases a rather reliable knowledge of nuclear cross-sections arrived at by a detalled evaluation of all the experimental dat are needed. This could be supplied by the various evaluations in the ENDF/B Library, whose contents and erphasis have been mainly determined by the needs of the thermal and fant reactor research prograus. In sove other cases larger uncertainties In the cross-sections could be colerated because all that one needs are order of-aigntude estimuter. In the later case, one could use partial evaluations of the various nuclei where the data files are ande up of a few reactions of ingediate incerest and the data files have not been tested againat integral measurements. In addition, there are a fet nuclei whose cross-gectiona have not been measured efther because they are unstable or because of orher special expertmental difficulties. In such cases, the cross-sections have to be estinated from calculations based on nuclear wodels and or nuclear syatenatics. In satisfying requeats for nuclear data for the CTR program, material obtained fron these different sources or procedures has been used and these wethods are described below. 


\subsection{ENDE/B LL1brary}

The origin and the general philosphy of this neutron cross-section data library have been described.' It 18 meant to be a reference data library based on the best avallable microscopic experimental data and covers a neutron energy range from $1.0 \mathrm{E}-05 \mathrm{eV}$ to $20.0 \mathrm{HeV}$. Though the earlier versions of this library contalned only neutron cross-section data, Verstons III and IV (the current version released a.b.-Sept. 74) also contain gamba production cross-Bections for a few nuclel used as shielding materials. As mentioned earlier, since this data library was formed with the needs of thermal and fast reactor applications in aind, the energy region below a few MeV has perhaps recelved more emphasis than the higher energy at around $14 \mathrm{MeV}$ which 18 of interest to CTR applications. This has been partly due to the fact that there are more rellable and larger number of measurements at lower energles than at higher energles except for the region in the Immediate vicinity of $14 \mathrm{HeV}$. In addition, many of the evaluat lons are for an element ratier than its Individual lsotopes; thus creating special problems in energy deposition and charged particle production calculations. However, attempts are being made to improve the data fj.les in the Ligh energy region guided by relevant integral measurements and to give isotopic inforwation in the data flles. In addition, the ENDF/B data flles are well documented ${ }^{3)}$ and have benefited from the results of benchmark experiment8. Therefore, t1ll evaluations specifically meant for CTR applications become avallable the data files in the ENDF/B system are the best available. As a result of this, in satisfying requests for the CTR progrems the current ENDF/B bibrary list is scanned first and if the elements or 1sotopes whose cross-sectlons are requested are in *In Jan. 1974, MHCSc received the ENDL avaluations which emphastze the high energy regton of CTR Interest and which in some cases have been compared against pulsed sphere exper inents ${ }^{24}$. 
the library, the corresponding data files are sent. The list of evaluations in the ENDF/B-IV Library are gtven in AppendLx $A$.

\subsection{EEDF/A Libricy}

Th1s data library consiats of (1) earlier evaluations no longer in current use (1i) partial evaluations which deal wich only a feu reactions or (1it) complete or partial evaluations from other data librarles. As far as possible atteapts are ande to convert these date files to conform to the ENDR/B format, chough in some cases they do not. In the latter ease, they usll have to be modified for use with the usul ENDF/B procesaing codes. In addition, the provenunce of the evaluations in the ENbF/A Library asy not be known. However, son of thase evaluations could be of use for prelininary calculation and feabibility studiee. Therefore, if the evaluation for a particular nucleus is not found in the ENDF/B library, the contents of the ENDF/A Lbrary are searched. In adultion to the earliat evaluations, the ENDF/A library contains the following:

(1) Uxwpl. (Vereton 3) (Hatced Kingdon) Received July 2, 1973 General eross-gection data sets. There is no documencation on the data files; there to only an index listing the variou reuctions in the date files. It is cicled "CODN tadex to the Alderanston Nuclear Data Library Verslon: Harch 1973". EnDP forant

(2) UKHDL (Uersion 2) (United Kingdon) Received Hay 5, 2971 General cross-section data sets. Ref 4 . ENDP fornat

(3) E⿰氵工pL (U.S.A.) Recelved Jan. 25, 1974

The Larrence Livernore Laboratory Evaluated Nuclear Data Library (ENDL) translated Into ENDF/B format. It Includes photon product ton cross-sections. Ref 5. ENDE format

(4) STANDARDS (U,S,A.) Received Jan. 25, 1973 ENDF/B-III cross-section weasurenent standards, Ref 6. UNDP format 
(5) CSN (France) Received August 7, 1973

Cospliation of properties of fission product auclei. Ref 7. ENDF format

(6) SPENG (Sweden) Received Jan. 5, 1973

General cross-section data oets. This library wich is in part

based on UKNDL, ENDF and KEDAK Libraries contalis data for a number

of materials not in ENDF/B-III. Ref 8

(7) KEDAK (W. Germany) Received October 26, 1970

General cross-section data sets. Ref 9

(8) SAMD-II (U.S.A.) Received August 5, 1971

Dosimetry cross-sections. Ref 10

(9) MAEC (Australia) Received Sept. 15, 1971

Also referred to as "Cook Library" cross-sections for fisston

product nuclides. Ref 11. ENDF format

(10) OBNINSK (Nikolaev) (U.S.S.R.) Received May 30, 1974

Elastic Scattering and Legendre Coefficient fits to some 42

nuclides. No reference, ENDF format.

(11) KOYSHIN G NIKOLAPV (U.S.S.R.) Recelved Jan. 17, 1973

Fission cross-gection for ${ }^{235} \mathrm{U}$, Ref 23 . ENDF format

(12) BOYAD (U.S.S,R.) Received Sepc, 11, 1972

$238 \mathrm{u}$ evaluation. No reference.

(13) BENZ1 (Italy) Recelved Dec, 4, 1970

Faat neutron radiative capture crosg-sections of nuclet. Ref 12.

(14) LIVOLSI 1500 (U.S.A.) Received Nov. 1, 1971

These are based on ti: cook 1ibrary, with sove 1mprovements and

modifications in the thermal and resonance reglons. Ref 13. ENDF format

Furcher information on some of these data libreries may be found In Ref 14.

A parcial lioting of the nuclides and their material numbers for

identification may be found in Appendix B. 


\subsection{Calculations Based on Nuclear Systematics}

These calculations are performed by using tile code THREst which has been described in detall. This code calculates some nineteen (n,particle) cross-sectlons lnduced by neutrons beiow $20.0 \mathrm{MeV}$ in medium mass nuclel. The crosg-sections for charged particle reactions as well as fission spectrun averages of the cross-sections can be calculated.

This code describes a particular cross-section and its varlation with energy by a form given by the statistical model. Further, the varfous parameters such as peak hefght, its width, steepness with which It rises or falls are deseribed by a set of parameterg which are determined by existing experimental data and their variation for nuclel with different 2 and $N$. Thus the only inputs needed for the code are the $2, N$ of the target nucleus and the Q-values for some nineteen nuclear reactions wich could be calculated from the recent mass tables of Wapstra and Gove or from an approximate mass formula buitt into the program. The reactions for which the cross-sections are calculated are $\left(n, n^{\prime}\right),(n, 2 n),(n, 3 n)$, $(n, p),(n, d),(n, t),\left(n,{ }^{3} H e\right),(n, \alpha),(n, n p),(n, n d),(n, n t),\left(n, n^{3} H e\right)$, $(n, n \alpha),(n, 2 p),(n, p \alpha),(n, d n),(n, \alpha n)$, and $(n, \alpha p)$. The code Thresh is befing constantly updated to Include the latest information on experimental data in the form of gome 13 parameters in terms of which the cross-sections are calculated. Recently, it has also been extended to heavier nuclei to Include the mass region up to $A=83$. In addition, stratlar procedures have been adopted in a code CHAPIN to calculate charged particle crosssections. The calculated cross-sections are glven both in the form of a line-printer output as well as in the standard ENDF/B format from which they can be plotted or can be processed further.

It $1 \mathrm{~s}$ interesting to compare the results of this cade with avallable experimental data. This is shown in Fig. 1 where the experimental data on the ${ }^{58} \mathrm{~N}_{1}(\mathrm{n}, 2 \mathrm{2})$ cross-section are shown from the threshold of the reaction 
at $12.415 \mathrm{MeV}$ to $20.0 \mathrm{HeV}$. Some of the avallable data sets have been left out as they were chought to be highly discrepant or fear of cluctering up the figure with too many points. The continuous curve drawn through the experimental points is thought to be the best representation of the available experinental daca. The dashed curve shows the result of 'HRESH caiculations. The average ratio of the values on the continuous curve to the corresponding ones on the dashed curve is 0.74 with the minimum and the maximum ratios in the whole energy range differing by only $10 \%$.

The problems assoclated with the $(n, 2 n)$ cross-section of ${ }^{93} \mathrm{Nb}$ i! another cage where THRESH type calculations baged on nuelear systematics have proved to be helpful. The activation measurements of the ${ }^{93} \mathrm{Nb}(n, 2 n)$ cross-section gave a value of about $450 \mathrm{mb}$ at $14 \mathrm{HeV}$ whereas nuclear gystematics predicted (18) a cross-section of $1281 \mathrm{ab}$ at $34.1 \mathrm{KeV}$. This problam was solved ${ }^{9)}$ with the realiaation that the: ground state of ${ }^{92} \mathrm{Nb}$ populated in the $(n, 2 n)$ reection has a very long helf-Iffe and that the aeasured activity corresponds to cransitions to the first excited atate with a relatively short half-life. Subsequently, experiments designed to measure the outgolng neutrons and the $(n, 2 n)$ cross-sections thus weasured have given results in subatantial agreenent with the ThPESH type calculations. Thus Mather et $(20)$ aeasured $1,312 \pm 83 \mathrm{ab}$ at $14.3 \mathrm{MeV}$ and Huring et $\mathrm{II}^{2}$ obtained $1350 \pm 250 \mathrm{mb}$ at $14 \mathrm{MeV}$ and Paulsen and Wider: ${ }^{(22)}$ got $1380 \pm 179$ wb at $16.8 \mathrm{keV}$. However, these examples should not be construed to mean that the results of THRESH calculations for all the (n,particle) reactions for all the mediun mast nuclei agree so well with experimental data - the differences between them are usually much larger. Therefore, in those cased where there are no experimental data, this code can supply cross-aection values which are sonetimes moderately reliable or at least can be trusted to glve order-of-wagnitude estinntes. 


\section{List of CTR Requests}

There have been mainly three sets of requests for nuclear data for CTR related programs. They are by (1) C. W. Maynard of Wiscons in University for energy desposition, charged particle production and radioactivity calculations (2) J. R. Powell of Brookhaven in connection with his studies of the minimum activity blankets and (3) W. C. Wolkenhower for his calcuIations of the fission-product burner systems. The detailed lists of these requests and the different data sets sent in June - August 1973 are given In Appendix c. In each case, either the available evaluations and or the results of the THREsk calculattons were sent to the requestar along with the plots of all the cross-sections. As such the data packages sent were voluminous and therefore are not given as part of this report. These data sets were also sent to some eight laboratories in the U.S. actively working in the CTR relsi.ed programs, Shallar requests for cross-seetions can be satisfied in the fucure. 
APPENDIX A

Contents of the ENDE/B-IV Library

$-9-$ 


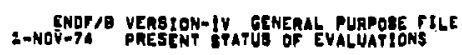

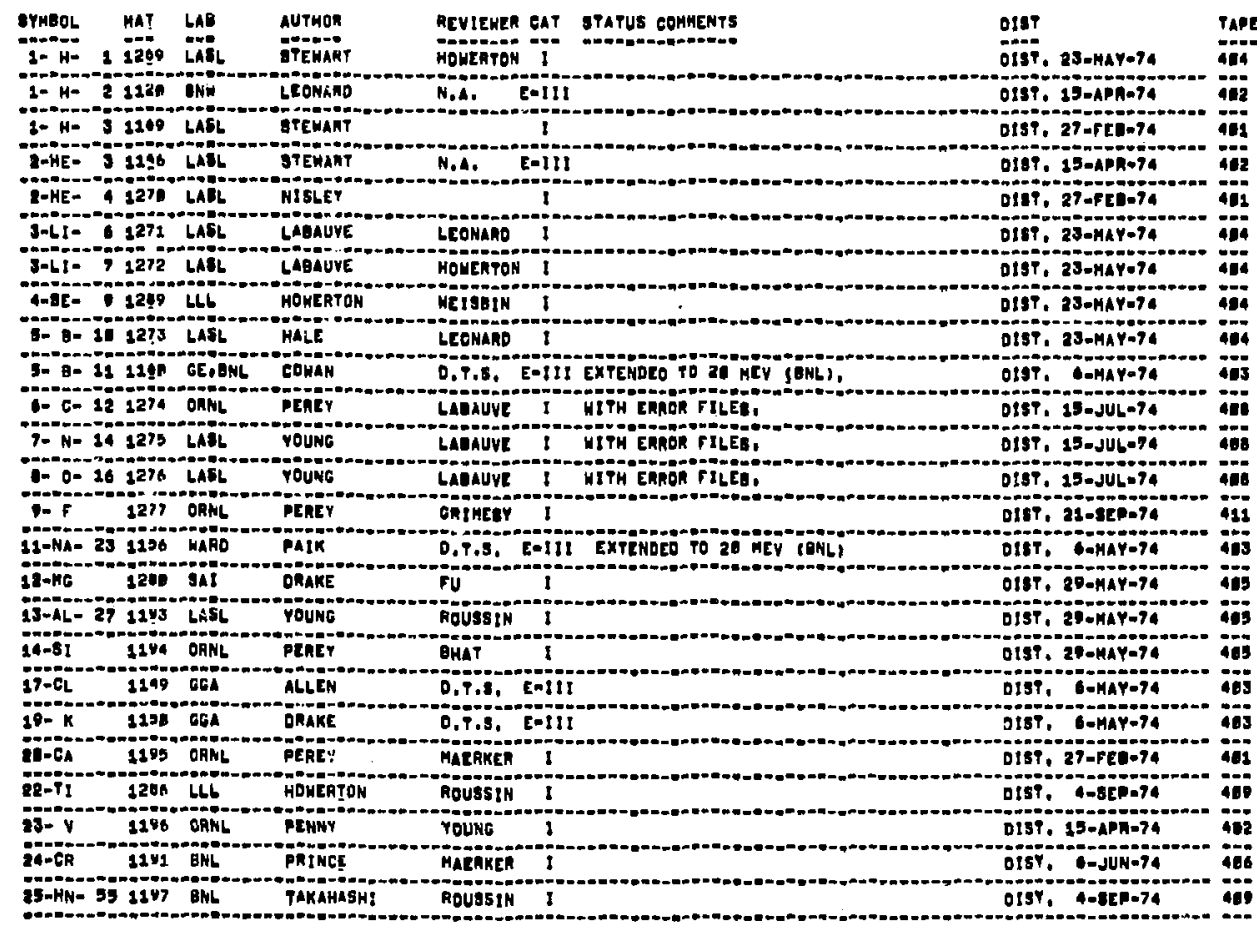




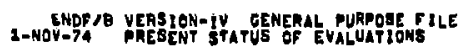

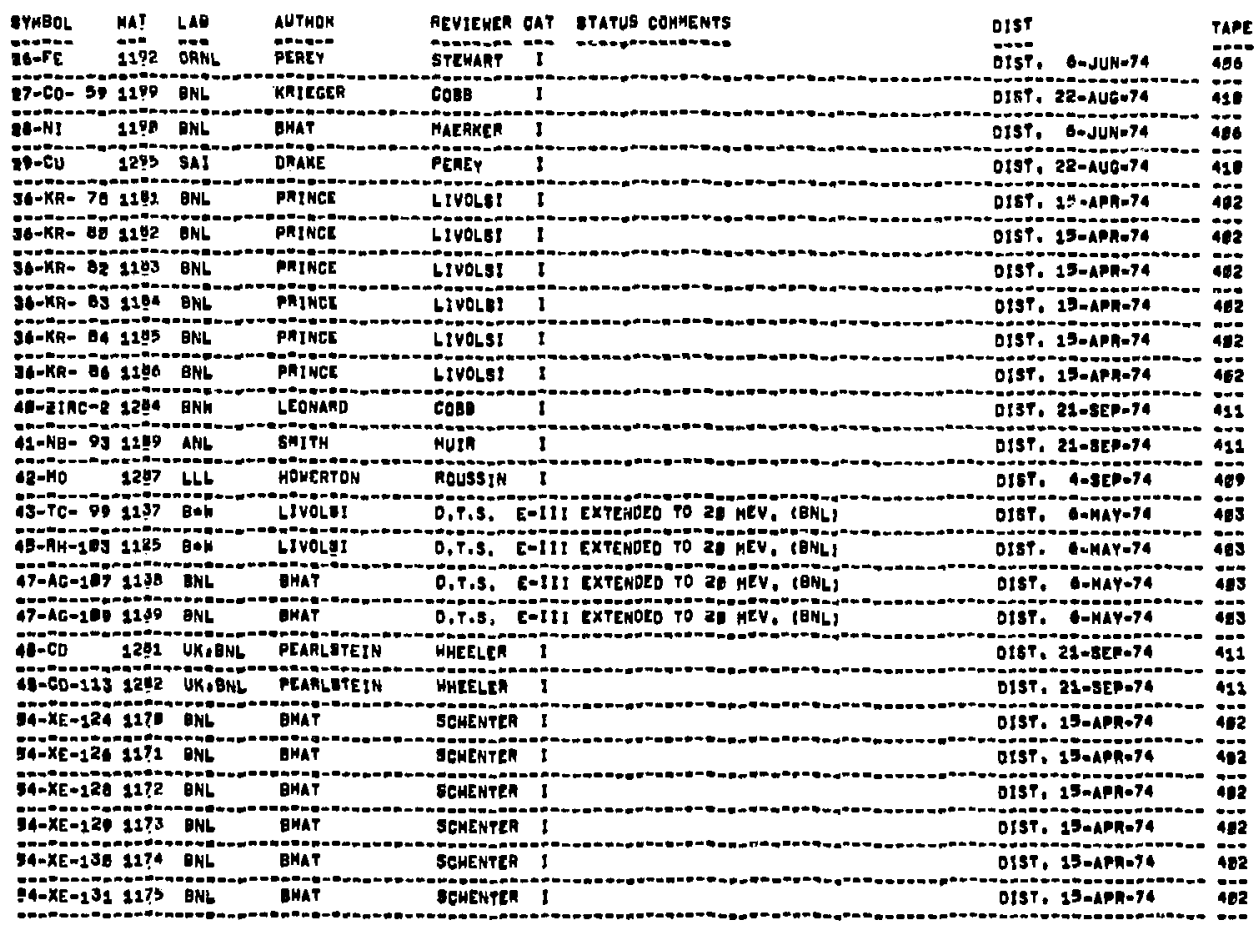




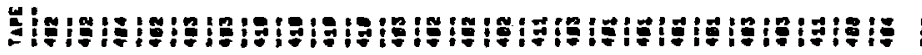
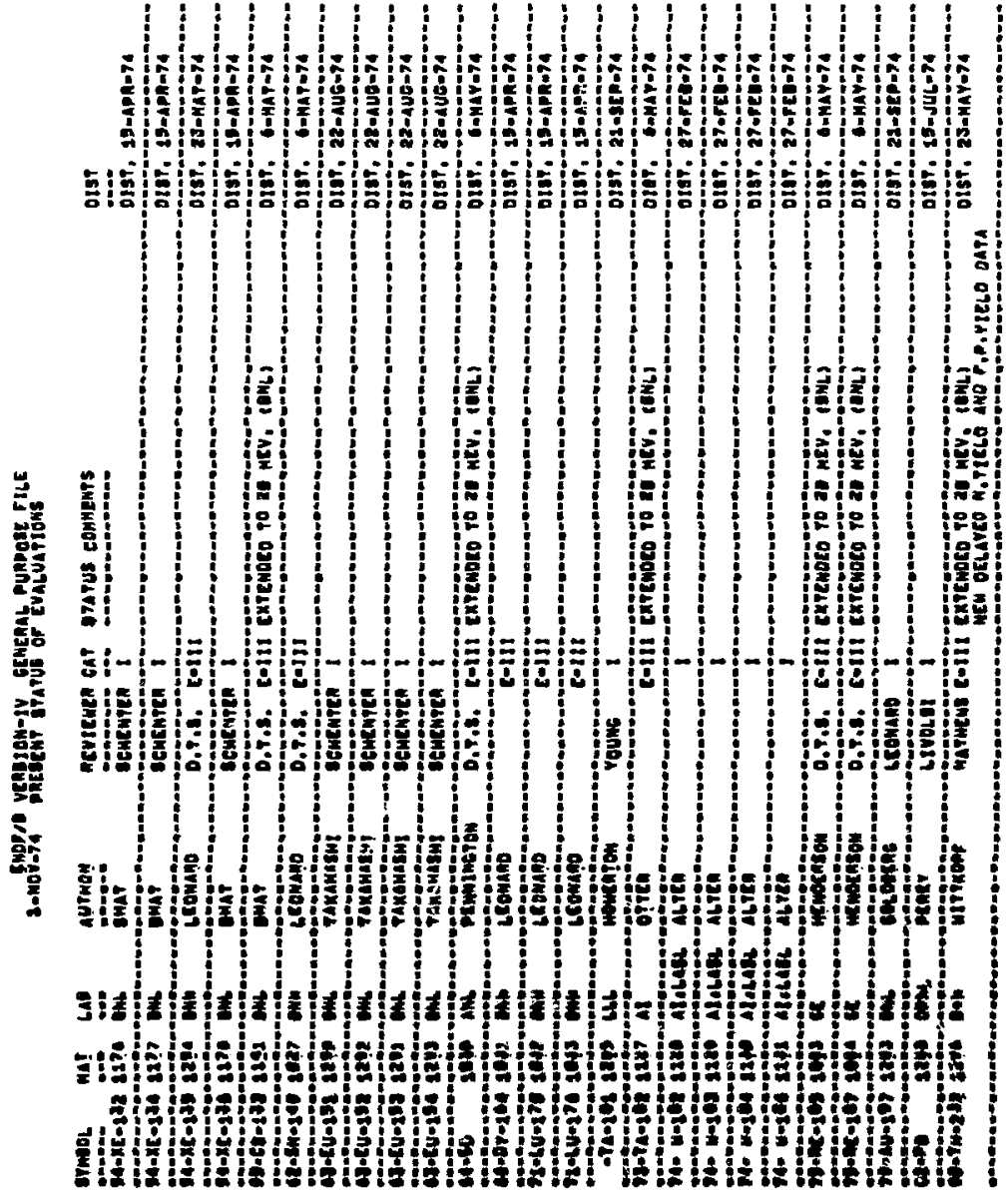


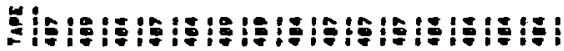

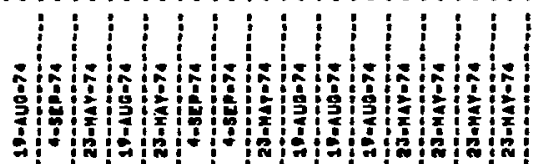

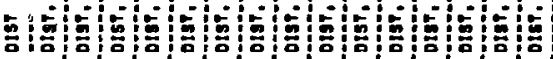

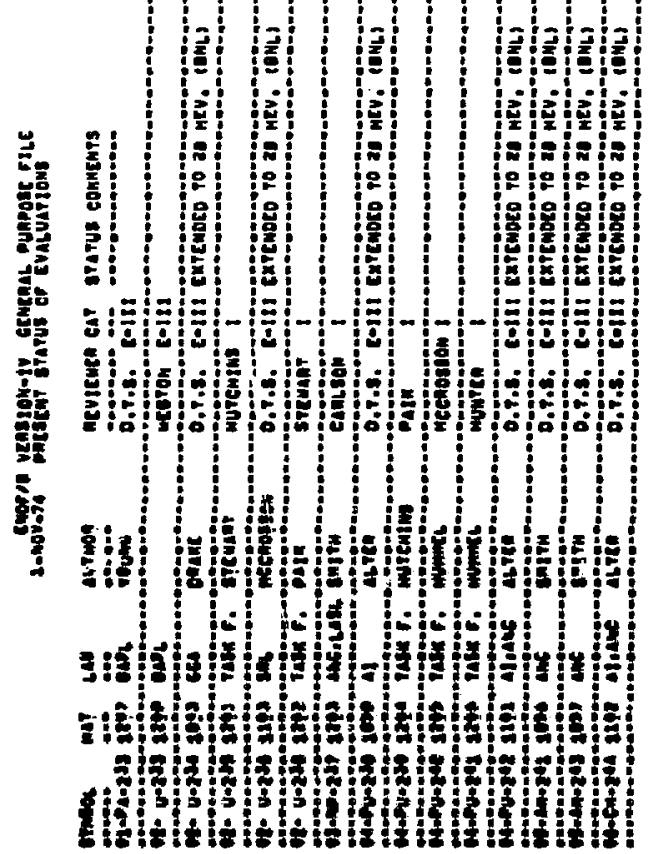


APPENDIX B

Partial Contents of the ENDF/A Libraxy

$-15-$

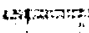


ENDF/A-701

AAEC Fiosion Product Data Llbrary Recelved 9/15/71 Cook Library

\begin{tabular}{|c|c|c|c|}
\hline Isotope* & $\underset{*}{\operatorname{AAEC}}$ & $\begin{array}{c}\text { ENDF } \\
\text { FOFUAT } \\
\text { MAT } \\
\vdots\end{array}$ & $\begin{array}{l}\text { \# OF } \\
\text { RECORDS } \\
\text { ENDP } \\
\text { EORMAT }\end{array}$ \\
\hline $2 n-72$ & 1 & 7001 & 483 \\
\hline Ga-72 & 2 & 7002 & $"$ \\
\hline$G t-72$ & 3 & 7003 & $"$ \\
\hline$G e-73$ & 4 & 7004 & $"$ \\
\hline Ge-74 & 5 & 7005 & $"$ \\
\hline Ge-76 & 6 & 7006 & " \\
\hline Ge-77 & 7 & 7007 & " \\
\hline$A B-75$ & $\mathbf{B}$ & 7008 & 11 \\
\hline$A s-76$ & 9 & 7009 & " \\
\hline As -77 & 10 & 7010 & " \\
\hline Se-76 & 11 & 7011 & " \\
\hline se-77 & 12 & 7012 & " \\
\hline $\mathrm{Se}-7 \mathrm{~B}$ & 13 & 7013 & " \\
\hline Se-79 & 14 & 7014 & $"$ \\
\hline $\operatorname{Se}-80$ & 15 & 7015 & $"$ \\
\hline$B r-81$ & 16 & 7016 & $"$ \\
\hline Br -82 & 17 & 7017 & $"$ \\
\hline $\mathrm{Kr}-82$ & 18 & 7018 & $"$ \\
\hline$K x-83$ & 19 & 7019 & $"$ \\
\hline$K T-84$ & 20 & 7020 & $"$ \\
\hline$k r-85$ & 21 & 7021 & $" 1$ \\
\hline $\mathrm{Kr}-86$ & 22 & 7022 & $"$ \\
\hline$R b-85$ & 23 & 7023 & $"$ \\
\hline$R b-86$ & 24 & 7024 & " \\
\hline$R b-87$ & 25 & 7025 & $"$ \\
\hline $5 r-86$ & 26 & 7026 & 495 \\
\hline$S T-88$ & 27 & 7027 & 483 \\
\hline
\end{tabular}


ENDF/A-701 (cont'd)

\begin{tabular}{|c|c|c|c|}
\hline Isotope * & $\begin{array}{c}\text { AAEC } \\
\text { 非 }\end{array}$ & $\begin{array}{c}\text { ENDF } \\
\text { FORMAT } \\
\text { MAT } \\
\#\end{array}$ & $\begin{array}{l}\text { \# OF } \\
\text { RECORDS } \\
\text { ENDF } \\
\text { FORMAT }\end{array}$ \\
\hline Sr-89 & 28 & 7028 & 483 \\
\hline Sr -90 & 29 & 7029 & $"$ \\
\hline Sr-91 & 30 & 7030 & " \\
\hline$Y-89$ & 31 & 7031 & $"$ \\
\hline $\mathbf{Y}-90$ & 32 & 7032 & $"$ \\
\hline$Y-91$ & 33 & 7033 & $"$ \\
\hline$Y-93$ & 34 & 7034 & " \\
\hline $2 x-90$ & 35 & 7035 & $"$ \\
\hline Zr-91 & 36 & 7036 & 489 \\
\hline $\mathrm{Zr}-92$ & 37 & 7037 & 501 \\
\hline $2 r-93$ & 38 & 7038 & 483 \\
\hline \multirow[t]{2}{*}{$2 r-94$} & 39 & 7039 & 501 \\
\hline & & & 8,891 \\
\hline
\end{tabular}

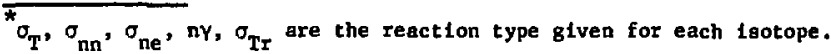


ENDF/A-702

AAEC F1ssion Product Data Library Received 9/15/71 Cook Library

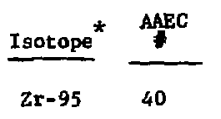

ENDF

EORMAT

MAT

$\#$

7040

\# $\mathrm{OF}$

$96 \quad 41$

7041

RECORDS

$97 \quad 42$

7042

Nb-95 43

7043

No-95 44

7044

7045

7046

7047

$\begin{array}{ll}98 & 47\end{array}$

7048

$99 \quad 48$

7049

Tc-99 50

7050

Ru-100 51

7051

7052

10152

7053

7054

$103 \quad 54$

7055

7056

$105 \quad 56$

7057

Rh-103 58

7058

$105 \quad 59$

7059

Pd-104 60

7060

7061

105

61

7062

$107 \quad 63$

7063

108 64

7054

10965

7065

$110 \quad 66$

7066

112

67

7067

Ag- $109 \quad 68$

7068

7069

ENDF

FORMAT

11169

(cont'd) 
ENDF/A-702 (cont'd)

\begin{tabular}{|c|c|c|c|}
\hline Isotope $^{*}$ & $\begin{array}{c}\text { AAEC } \\
\#\end{array}$ & $\begin{array}{c}\text { ENDF } \\
\text { FORMAT } \\
\text { MAT } \\
\#\end{array}$ & $\begin{array}{l}\text { \# OF } \\
\text { RECORDS } \\
\text { ENDF } \\
\text { FORMAT }\end{array}$ \\
\hline $\mathrm{Cd}-110$ & 70 & 7070 & 483 \\
\hline 111 & 71 & 7071 & " \\
\hline 112 & 72 & 7072 & $"$ \\
\hline 113 & 73 & 7073 & $"$ \\
\hline 114 & 74 & 7074 & " \\
\hline 115 & 75 & 7075 & $"$ \\
\hline 116 & 76 & 7076 & $"$ \\
\hline In- 115 & 77 & 7077 & tr \\
\hline Sn-115 & 78 & 7078 & $"$ \\
\hline
\end{tabular}

$\overline{18,843}$ TOTAL

$\bar{\sigma}_{\mathrm{T}}, \sigma_{\mathrm{nn}}, \sigma_{\mathrm{ne}}, \mathrm{ny}, \sigma_{\mathrm{Tr}}$ are the reaccion type given for each lsotope. 
ENDF/A-703

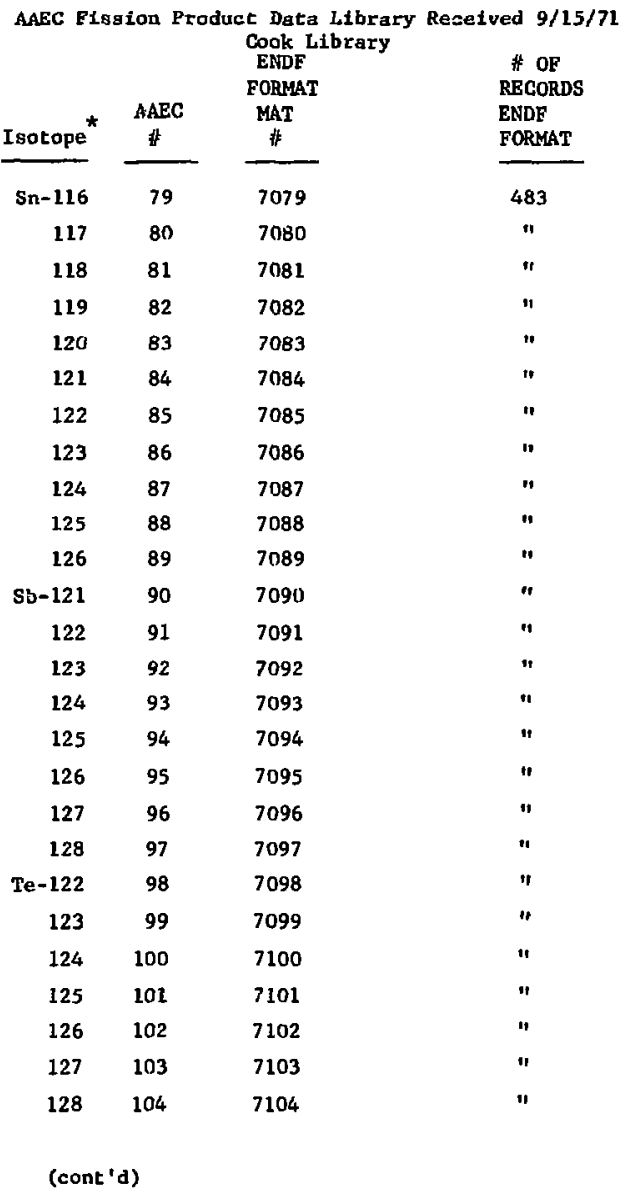


ENDF/A-703 (cont'd)

\begin{tabular}{|c|c|c|c|}
\hline Isotope* & $\underset{\forall}{\operatorname{AAEC}}$ & $\begin{array}{c}\text { ENDF } \\
\text { FORMAT } \\
\text { MAT } \\
\forall\end{array}$ & $\begin{array}{l}\text { Af OF } \\
\text { RECORDS } \\
\text { ENDE } \\
\text { FORMAT }\end{array}$ \\
\hline $\mathrm{Te}-129$ & 105 & 7105 & 483 \\
\hline 130 & 106 & 7106 & $"$ \\
\hline 131 & 107 & 7107 & $"$ \\
\hline 132 & 108 & 7108 & $"$ \\
\hline$I-127$ & 109 & 7109 & " \\
\hline 129 & 110 & 7110 & $"$ \\
\hline 130 & 111 & 7111 & " \\
\hline 131 & 112 & 7112 & " \\
\hline 133 & 113 & 7113 & " \\
\hline 135 & 114 & 7114 & $" 1$ \\
\hline $\mathrm{X} e-128$ & 115 & 7115 & " \\
\hline 130 & 116 & 7116 & $"$ \\
\hline \multirow[t]{2}{*}{131} & 117 & 7117 & $"$ \\
\hline & & & 18,837 \\
\hline
\end{tabular}

${ }^{*} \sigma_{T}, \sigma_{n n}, \sigma_{n e}, n Y, \sigma_{T r}$ are the reaction type given for each isotope. 
ENDF/A -704

AAEC Fission Product Data Ltbrary Received 9/15/71 Cook Llbrary

ENDF \# OF

\begin{tabular}{|c|c|c|}
\hline Isctope * & $\underset{\forall}{\operatorname{AAEC}}$ & $\underset{*}{\text { MAT }}$ \\
\hline$x e-132$ & 118 & 7118 \\
\hline 133 & 119 & 7119 \\
\hline
\end{tabular}

RECORDS

$134 \quad 120 \quad 7120$

$135 \quad 121 \quad 7121$

$\begin{array}{lll}136 & 122 & 7122\end{array}$

Cs $-133 \quad 123 \quad 7123$

$134 \quad 124 \quad 7124$

$135 \quad 125 \quad 7225$

$136 \quad 126 \quad 7126$

13, $127 \quad 7127$

Ba- $134 \quad 128 \quad 7128$

$\begin{array}{lll}136 & 129 & 7129\end{array}$

$137 \quad 130 \quad 7130$

$138 \quad 131 \quad 7131$

$140 \quad 132 \quad 7132$

La=139 $133 \quad 7133$

$140 \quad 134 \quad 7134$

Ce $-140 \quad 135 \quad 7135$

$141 \quad 136 \quad 7136$

$142 \quad 137 \quad 7137$

$143 \quad 138 \quad 7138$

$144 \quad 139 \quad 7139$

Pr-141 $140 \quad 7140$

$\begin{array}{lll}142 & 141 & 7141 \\ 143 & 142 & 7142\end{array}$

$145 \quad 143 \quad 7143$

(cont'd) 
ENDF/A -704 (cont'd)

\begin{tabular}{|c|c|c|c|}
\hline I so tope* & YEC & $\begin{array}{c}\text { ENDF } \\
\text { FORMAT } \\
\text { MAT } \\
\$\end{array}$ & $\begin{array}{l}\text { \# OF } \\
\text { RECORDS } \\
\text { ENDF } \\
\text { FORHAT }\end{array}$ \\
\hline Nd- 142 & 144 & 3144 & 483 \\
\hline 143 & 145 & 3145 & 1 \\
\hline 144 & 146 & 7146 & $"$ \\
\hline 145 & 147 & 7147 & $"$ \\
\hline 146 & 148 & 7148 & " \\
\hline 143 & 149 & 7149 & " \\
\hline 148 & 150 & 7150 & " \\
\hline 150 & 151 & 7151 & $"$ \\
\hline$P a-147$ & 152 & 7152 & $n$ \\
\hline 148 & 153 & 7153 & " \\
\hline 149 & 154 & 7154 & $"$ \\
\hline 151 & 155 & 7155 & $"$ \\
\hline \multirow[t]{2}{*}{$5 m-147$} & 156 & 7156 & 1 \\
\hline & & & 18,843 \\
\hline
\end{tabular}

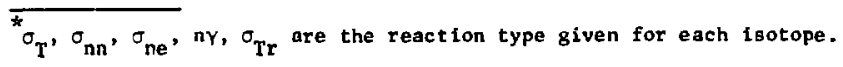


ENDF/A $=705$

AAEC Fission Product Data Library Received 9/15/71 Cook Library

\begin{tabular}{|c|c|c|c|}
\hline Isotope* & $\begin{array}{c}\text { AAEC } \\
\#\end{array}$ & $\begin{array}{c}\text { ENDF } \\
\text { FORMAT } \\
\text { MAT } \\
\forall\end{array}$ & $\begin{array}{l}\text { \# OF } \\
\text { RECORDS } \\
\text { ENDF } \\
\text { FORMAT }\end{array}$ \\
\hline$S m-148$ & 157 & 7157 & 483 \\
\hline 149 & 158 & 7158 & $"$ \\
\hline 150 & 159 & 7159 & $"$ \\
\hline 151 & 160 & 7160 & $"$ \\
\hline 152 & 161 & 7161 & $n$ \\
\hline 153 & 162 & 7162 & $"$ \\
\hline 154 & 163 & 7163 & " \\
\hline 156 & 164 & 7164 & $" \prime$ \\
\hline Eu-153 & 165 & 7165 & $"$ \\
\hline 154 & 166 & 7166 & $"$ \\
\hline 155 & 167 & 7167 & " \\
\hline 156 & 168 & 7168 & $"$ \\
\hline 157 & 169 & 7169 & $"$ \\
\hline$G d-155$ & 170 & 7170 & $"$ \\
\hline 156 & $1 \div 1$ & 7171 & " \\
\hline 157 & 712 & 7172 & $"$ \\
\hline 158 & 173 & 7173 & "r \\
\hline 159 & 174 & 7174 & $"$ \\
\hline 160 & 175 & 7175 & $"$ \\
\hline$T b-159$ & 176 & 7176 & $n$ \\
\hline 160 & $1 ; 7$ & 7177 & $"$ \\
\hline 161 & 178 & 7178 & " \\
\hline$D y-160$ & 179 & 7179 & $"$ \\
\hline 161 & 180 & 7180 & $"$ \\
\hline
\end{tabular}


ENDF/A-705 (cont ${ }^{\prime} d$ )

\begin{tabular}{|c|c|c|c|}
\hline Isotope ${ }^{*}$ & $\begin{array}{c}\text { AAEC } \\
\|\end{array}$ & $\begin{array}{c}\text { ENDF } \\
\text { EORKAT } \\
\text { MAT } \\
\text { \# }\end{array}$ & $\begin{array}{l}\text { \# OF } \\
\text { RECORDS } \\
\text { ENDE } \\
\text { FORMAT }\end{array}$ \\
\hline Dy-162 & 181 & 7181 & 483 \\
\hline 163 & 182 & 7182 & $n$ \\
\hline 164 & 183 & 7183 & $"$ \\
\hline $\mathrm{H}_{0}-165$ & 184 & 7184 & $"$ \\
\hline Tc-799 & 185 & 7185 & $"$ \\
\hline Cd-815 & 186 & 7186 & $"$ \\
\hline $\mathrm{Te}-823$ & 187 & 7187 & $"$ \\
\hline 825 & 188 & 7188 & $"$ \\
\hline 827 & 189 & 7189 & $"$ \\
\hline 829 & 190 & 7190 & $"$ \\
\hline 831 & 191 & 7191 & $n$ \\
\hline$P_{m}=848$ & 192 & 7192 & $"$ \\
\hline
\end{tabular}

17,388 TOTAL

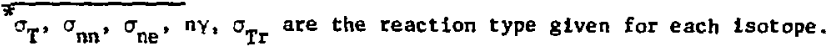


Bif

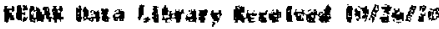

\begin{tabular}{|c|c|c|c|c|c|c|}
\hline \multirow[b]{2}{*}{ Intedn } & \multicolumn{5}{|c|}{ 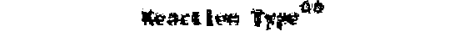 } & \multirow{2}{*}{ 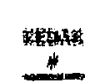 } \\
\hline & 5 & 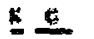 & 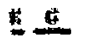 & 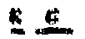 & 里 & \\
\hline $41=20$ & 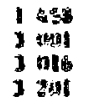 & 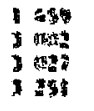 & 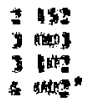 & $\begin{array}{l}4151 \\
1206 \\
7101\end{array}$ & 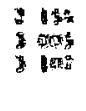 & $1 y \operatorname{lng}$ \\
\hline$c-12$ & 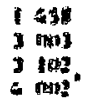 & 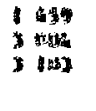 & $\begin{array}{l}3138 \\
2405 \\
2158\end{array}$ & 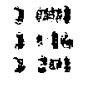 & 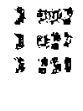 & 45 \\
\hline cd & $\begin{array}{l}1475 \\
1406 \\
7145\end{array}$ & 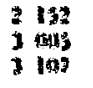 & 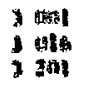 & 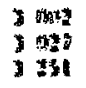 & $\begin{array}{l}3 \text { ats } \\
\$ \text { tat }\end{array}$ & \& $\log _{0}$ \\
\hline Cr & $\begin{array}{l}165 \\
3 \text { at } \\
3014 \\
3241 \\
3461\end{array}$ & 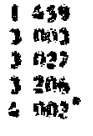 & 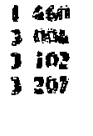 & $\begin{array}{l}2157 \\
34+13 \\
7109 \\
1251\end{array}$ & 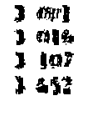 & 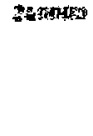 \\
\hline Cr-3n & 1458 & 1459 & $213 \pm$ & 2151 & $\geq 15 t$ & $29+250$ \\
\hline ce-sz & 1458 & 1550 & 2132 & $\geq 153$ & 215 & 2antost \\
\hline Cr-33 & 1458 & $1+53$ & 2152 & 3193 & $31 y$ & $24005 y$ \\
\hline$C x-56$ & 1658 & 1459 & 2152 & 2153 & 3154 & 360018 \\
\hline Fe & $\begin{array}{ll}1 & 458 \\
3 & 002 \\
3 & 019 \\
3 & 201 \\
3 & 461\end{array}$ & $\begin{array}{ll}1 & 459 \\
3 & 013 \\
3 & 427 \\
3 & 206 \\
4 & 002\end{array}$ & $\begin{array}{ll}1 & 460 \\
3 & 4646 \\
3 & 11: 2 \\
3 & 2117\end{array}$ & $\begin{array}{ll}2 & 152 \\
3 & 005 \\
3 & 103 \\
3 & 251\end{array}$ & $\begin{array}{l}3 \text { and } \\
3 \text { a16 } \\
3 \text { 107 } \\
3452\end{array}$ & 26 mPlan \\
\hline Fe-54 & 1458 & 1459 & 2152 & 2153 & 2156 & 261056 \\
\hline Fe-56 & 1458 & 1459 & 2152 & 2153 & 2154 & 26 nist \\
\hline Pe-ST & 1458 & 1459 & 2152 & 2153 & 2154 & 2611057 \\
\hline Fe-58 & 1458 & 1459 & 2154 & & & 260058 \\
\hline
\end{tabular}

(cont inued) 


\begin{tabular}{|c|c|c|c|c|c|c|}
\hline \multirow[b]{2}{*}{$1+1$} & \multirow{2}{*}{5} & \multicolumn{3}{|c|}{ 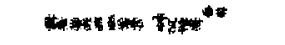 } & \multirow[b]{2}{*}{ \pm} & \multirow{2}{*}{ 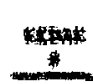 } \\
\hline & & $E$ & 4 & 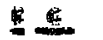 & & \\
\hline 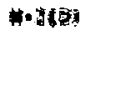 & 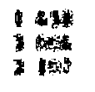 & $\begin{array}{l}18 \\
+14\end{array}$ & $\begin{array}{l}101 \\
04\end{array}$ & 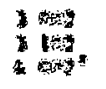 & 1 & 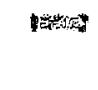 \\
\hline * & $\begin{array}{l}5 \\
584 \\
351\end{array}$ & 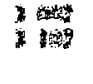 & 梳 & 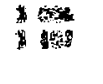 & 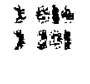 & 1todat \\
\hline II $H_{4}$ & $\begin{array}{l}y \\
y \\
y\end{array}$ & $\begin{array}{l}y \\
y\end{array}$ & 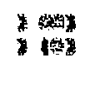 & 表 & 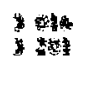 & 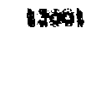 \\
\hline 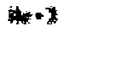 & 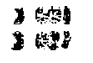 & tot & t to & $\begin{array}{l}y \\
1\end{array}$ & $\begin{array}{l}500 \\
y\end{array}$ & tots \\
\hline $4-\frac{1}{x^{2}}$ & $\begin{array}{l}140 \\
y\end{array}$ & 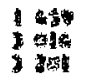 & 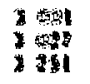 & 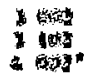 & 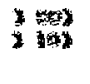 & 2 \\
\hline$\omega$ & 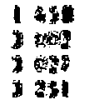 & 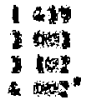 & $\begin{array}{l}14 \% \\
5 \% \\
10 \%\end{array}$ & $\begin{array}{l}z+2 \\
y+2 \\
y+2\end{array}$ & $\begin{array}{l}y \text { oft } \\
y+1\end{array}$ & 275009 \\
\hline$\omega *-\varphi_{2}$ & 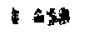 & (45) & 158 & 2131 & 215 & matst \\
\hline $4 n-2 x$ & 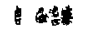 & $1+5 *$ & 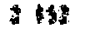 & $* 151$ & 24 & 4200 \\
\hline Na-ns & $1+14$ & 1412 & 15 & 75 & 714 & sansts \\
\hline $\sin 4$ & 1514 & 1 ast & 2138 & 132 & 314 & 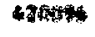 \\
\hline $\operatorname{mos}=\pi$ & 18511 & 1034 & 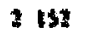 & 2153 & $: 13$ & tagant \\
\hline$N-4 t$ & $t 45$ & 164 & 3152 & 213 & $215=$ & $\operatorname{cons} 4$ \\
\hline$m-10 n$ & 140 & 1459 & 2152 & 213 & 2156 & 420100 \\
\hline . & $+n e^{\prime}$ & & & & & 200100 \\
\hline
\end{tabular}

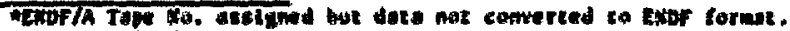

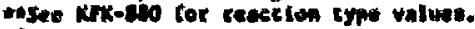

canker-at-rass syotem. 
ENDF/A-708*

KEDAK Data LIbrary Recelved 10/26/70

\begin{tabular}{|c|c|c|c|c|c|c|c|c|c|}
\hline \multirow[b]{2}{*}{ Isotope } & \multicolumn{8}{|c|}{ Reaction Type } & \multirow{2}{*}{$\begin{array}{c}\text { KEDAK } \\
*\end{array}$} \\
\hline & $\underline{K} G$ & $\underline{K} G$ & $\underline{x}$ & $\boldsymbol{G}$ & $\underline{k}$ & $G$ & $\underline{K}$ & $E$ & \\
\hline$N i$ & $\begin{array}{ll}1 & 458 \\
3 & 002 \\
3 & 019 \\
3 & 201 \\
3 & 461\end{array}$ & $\begin{array}{ll}1 & 459 \\
3 & 003 \\
3 & 027 \\
3 & 206 \\
4 & 002+\end{array}$ & $\begin{array}{ll}1 & 4 \\
3 & 0 \\
3 & 1 \\
3 & 2\end{array}$ & $\begin{array}{l}460 \\
004 \\
102 \\
207\end{array}$ & $\begin{array}{l}2 \\
3 \\
3 \\
3\end{array}$ & $\begin{array}{l}152 \\
005 \\
103 \\
25 t\end{array}$ & $\begin{array}{l}3 \\
3 \\
3 \\
3\end{array}$ & $\begin{array}{l}001 \\
016 \\
107 \\
452\end{array}$ & 280000 \\
\hline$N i=58$ & 1458 & 1459 & 21 & 152 & 2 & 153 & 2 & 154 & 280058 \\
\hline Si-60 & 1458 & 1459 & 21 & 152 & 21 & 153 & 2 & 154 & 280060 \\
\hline$M[-6 L$ & 1458 & 1459 & 21 & 154 & & & & & 280061 \\
\hline$N i-62$ & 1458 & 1459 & 21 & 152 & 21 & 154 & & & 280062 \\
\hline$N \in-64$ & 1458 & 1459 & 21 & 154 & & & & & 280064 \\
\hline $0-16$ & $\begin{array}{ll}1 & 458 \\
3 & 003 \\
3 & 107 \\
3 & 004\end{array}$ & $\begin{array}{ll}1 & 459 \\
3 & 005 \\
3 & 201 \\
3 & 102\end{array}$ & $\begin{array}{ll}2 & 1 \\
3 & 0 \\
3 & 2\end{array}$ & $\begin{array}{l}152 \\
016 \\
251\end{array}$ & $\begin{array}{l}30 \\
30 \\
40\end{array}$ & $\begin{array}{l}001 \\
027 \\
002+\end{array}$ & $\begin{array}{l}3 \\
3\end{array}$ & $\begin{array}{l}002 \\
103\end{array}$ & 80016 \\
\hline$v-235$ & $\begin{array}{ll}1 & 456 \\
2 & 153 \\
3 & 003 \\
3 & 027 \\
3 & 206 \\
4 & 002^{+}\end{array}$ & $\begin{array}{ll}1 & 457 \\
2 & 154 \\
3 & 004 \\
3 & 102 \\
3 & 207\end{array}$ & $\begin{array}{ll}1 & 4 \\
2 & 1 \\
3 & 0 \\
3 & 1 \\
3 & 2\end{array}$ & $\begin{array}{l}458 \\
155 \\
005 \\
103 \\
251\end{array}$ & $\begin{array}{ll}1 & 4 \\
3 & 0 \\
3 & 0 \\
3 & 1 \\
3 & 4\end{array}$ & $\begin{array}{l}459 \\
001 \\
016 \\
107 \\
452\end{array}$ & $\begin{array}{ll}2 & 1 \\
3 & 0 \\
3 & 0 \\
3 & 2 \\
3 & 4\end{array}$ & $\begin{array}{l}152 \\
002 \\
019 \\
201 \\
461\end{array}$ & 920235 \\
\hline
\end{tabular}

DENDF/A Tape No. assigned but data not converted to ENDF format. * SEe KFK-880 for reaction type values.

tCenter-of-Mass System. 
ENDF/A-709*

KEDAK Data Library Recetved 10/26/70

\begin{tabular}{|c|c|c|c|c|c|c|c|c|c|c|c|}
\hline \multirow[b]{2}{*}{ Isotope } & \multicolumn{10}{|c|}{ Reaction Type } & \multirow{2}{*}{$\begin{array}{c}\text { KEDAK } \\
\theta\end{array}$} \\
\hline & $\underline{\mathbf{x}}$ & $\mathbf{G}$ & $\underline{\mathbf{K}}$ & G & $\underline{\mathbf{k}}$ & G & $\underline{K}$ & G & $\kappa$ & 6 & \\
\hline $\mathrm{Na}-23$ & $\begin{array}{l}1 \\
3 \\
3 \\
3\end{array}$ & $\begin{array}{l}458 \\
001 \\
016 \\
201\end{array}$ & $\begin{array}{l}1 \\
3 \\
3 \\
3\end{array}$ & $\begin{array}{l}459 \\
002 \\
027 \\
251\end{array}$ & $\begin{array}{l}2 \\
3 \\
3 \\
4\end{array}$ & $\begin{array}{l}152 \\
003 \\
102 \\
002^{+}\end{array}$ & $\begin{array}{l}2 \\
3 \\
3\end{array}$ & $\begin{array}{l}153 \\
004 \\
103\end{array}$ & & $\begin{array}{l}154 \\
005 \\
107\end{array}$ & 110023 \\
\hline$P u-239$ & $\begin{array}{l}1 \\
2 \\
3 \\
3 \\
3 \\
4\end{array}$ & $\begin{array}{l}456 \\
153 \\
003 \\
027 \\
206 \\
0024\end{array}$ & $\begin{array}{l}1 \\
2 \\
3 \\
3 \\
3\end{array}$ & $\begin{array}{l}457 \\
154 \\
014 \\
102 \\
207\end{array}$ & & $\begin{array}{l}458 \\
155 \\
005 \\
103 \\
251\end{array}$ & $\begin{array}{l}1 \\
3 \\
3 \\
3 \\
3\end{array}$ & $\begin{array}{l}459 \\
001 \\
016 \\
107 \\
452\end{array}$ & $\begin{array}{l}2 \\
3 \\
3 \\
3 \\
3\end{array}$ & $\begin{array}{l}152 \\
002 \\
019 \\
201 \\
461\end{array}$ & 940239 \\
\hline Pu-240 & $\begin{array}{ll}1 & 4 \\
2 & 1 \\
3 & 0 \\
3 & 0 \\
3 & 2\end{array}$ & $\begin{array}{l}456 \\
153 \\
003 \\
027 \\
251\end{array}$ & $\begin{array}{l}1 \\
2 \\
3 \\
3 \\
3\end{array}$ & $\begin{array}{l}457 \\
154 \\
004 \\
102 \\
452\end{array}$ & $\begin{array}{l}1 \\
2 \\
3 \\
3 \\
4\end{array}$ & $\begin{array}{l}458 \\
155 \\
005 \\
201 \\
002^{+}\end{array}$ & $\begin{array}{l}1 \\
3 \\
3 \\
3\end{array}$ & $\begin{array}{l}459 \\
0111 \\
016 \\
206\end{array}$ & $\begin{array}{l}2 \\
3 \\
3 \\
3\end{array}$ & $\begin{array}{l}152 \\
002 \\
019 \\
207\end{array}$ & 940240 \\
\hline$P u-241$ & $\begin{array}{ll}1 & 1 \\
2 & 1 \\
3 & 0 \\
3 & 0 \\
3 & 2\end{array}$ & $\begin{array}{l}456 \\
153 \\
003 \\
027 \\
251\end{array}$ & $\begin{array}{l}1 \\
2 \\
3 \\
3 \\
3\end{array}$ & $\begin{array}{l}457 \\
154 \\
004 \\
102 \\
452\end{array}$ & $\begin{array}{l}1 \\
2 \\
3 \\
3 \\
4\end{array}$ & $\begin{array}{l}458 \\
155 \\
005 \\
201 \\
002^{+}\end{array}$ & $\begin{array}{l}1 \\
3 \\
3 \\
3\end{array}$ & $\begin{array}{l}459 \\
001 \\
016 \\
206\end{array}$ & $\begin{array}{l}2 \\
3 \\
3 \\
3\end{array}$ & $\begin{array}{l}152 \\
102 \\
019 \\
027\end{array}$ & 940241 \\
\hline $\mathrm{Pu}-242$ & $\begin{array}{ll}1 & 4 \\
2 & 1 \\
3 & 0 \\
3 & 1 \\
3 & 4\end{array}$ & $\begin{array}{l}456 \\
153 \\
004 \\
102 \\
452\end{array}$ & $\begin{array}{l}1 \\
2 \\
3 \\
3 \\
4\end{array}$ & $\begin{array}{l}457 \\
154 \\
005 \\
201 \\
002^{+}\end{array}$ & $\begin{array}{l}1 \\
3 \\
3 \\
3\end{array}$ & $\begin{array}{l}458 \\
001 \\
016 \\
206\end{array}$ & $\begin{array}{l}1 \\
3 \\
3 \\
3\end{array}$ & $\begin{array}{l}459 \\
002 \\
019 \\
207\end{array}$ & $\begin{array}{l}2 \\
3 \\
3 \\
3\end{array}$ & $\begin{array}{l}152 \\
003 \\
027 \\
251\end{array}$ & 940242 \\
\hline$v-238$ & $\begin{array}{ll}1 & 4 \\
2 & 1 \\
3 & 0 \\
3 & 1 \\
3 & 4\end{array}$ & $\begin{array}{l}456 \\
153 \\
004 \\
102 \\
452\end{array}$ & $\begin{array}{l}1 \\
2 \\
3 \\
3 \\
3\end{array}$ & $\begin{array}{l}457 \\
154 \\
005 \\
103 \\
461\end{array}$ & $\begin{array}{l}1 \\
3 \\
3 \\
3 \\
4\end{array}$ & $\begin{array}{l}458 \\
001 \\
016 \\
107 \\
002^{+}\end{array}$ & $\begin{array}{l}1 \\
3 \\
3 \\
3\end{array}$ & $\begin{array}{l}459 \\
002 \\
019 \\
201\end{array}$ & $\begin{array}{l}2 \\
3 \\
3 \\
3\end{array}$ & $\begin{array}{l}152 \\
003 \\
027 \\
251\end{array}$ & 920238 \\
\hline
\end{tabular}

\#ENDF/A Tape No. assigend but data not converted to ENDF format. * See KFK-880 for reaction type values

+Center-of-Mass System. 


\section{CHoF/A-60!}

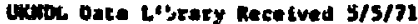
Version 2

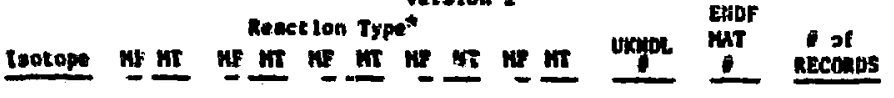

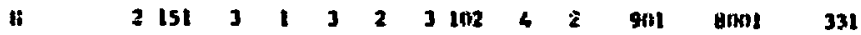

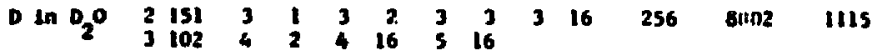

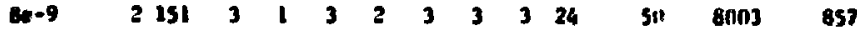
$\begin{array}{lllllllllllll}3 & 102 & 3 & \text { ins } & 3 & 102 & 4 & 2 & 4 & 24\end{array}$

$\begin{array}{rrrrrrrrrrrrrr}C .12 & 2 & 151 & 3 & 1 & 3 & 2 & 3 & 3 & 3 & 23 & 60 & 8004 & 1904 \\ & 3 & 51 & 3 & 102 & 3 & 107 & 4 & 2 & 4 & 23 & & \end{array}$ 4 SI 523

$0 \cdot 16$

$\begin{array}{rrrrrrrrrr}2 & 151 & 3 & 1 & 3 & 2 & 3 & 3 & 3 & 4 \\ 3 & 51 & 3 & 52 & 3 & 54 & 3 & 55 & 3 & 57 \\ 3 & 58 & 3 & 59 & 3 & 61 & 3 & 91 & 3 & 102 \\ 3 & 103 & 3 & 104 & & & 3 & 107 & 4 & 2 \\ 4 & 51 & 4 & 52 & 4 & 53 & 4 & 54 & 4 & 55 \\ 4 & 56 & 4 & 57 & 4 & 58 & 4 & 59 & 4 & 60 \\ 4 & 91 & 5 & 91 & 3 & 53 & 3 & 56 & & \end{array}$

$\begin{array}{lllllllllll}1-235 & 2 & 151 & 3 & 1 & 3 & 2 & 3 & 3 & 3 & 16\end{array}$ $\begin{array}{llllllllll}3 & 17 & 3 & 18 & 3 & 51 & 3 & 52 & 3 & 53 \\ 3 & 54 & 3 & 55 & 3 & 56 & 3 & 91 & 3 & 102\end{array}$ $\begin{array}{llllllllll}4 & 2 & 4 & 16 & 4 & 17 & 4 & 18 & 4 & 51\end{array}$ $\begin{array}{llllllllll}4 & 52 & 4 & 53 & 4 & 54 & 4 & 55 & 4 & 56\end{array}$ $\begin{array}{llllllllll}4 & 91 & 5 & 16 & 5 & 17 & 5 & 18 & 5 & 91\end{array}$

1452

Pu-239

$\begin{array}{llllllllll}2 & 151 & 3 & 1 & 3 & 2 & 3 & 3 & 3 & 16\end{array}$

$\begin{array}{llllllllll}3 & 17 & 3 & 18 & 3 & 51 & 3 & 52 & 3 & 53\end{array}$

$\begin{array}{llllllllll}3 & 54 & 3 & 55 & 3 & 36 & 3 & 57 & 3 & 91\end{array}$

$\begin{array}{llllllllll}3 & 102 & 4 & 2 & 4 & 16 & 4 & 17 & 4 & 18\end{array}$

$\begin{array}{llllllllll}4 & 51 & 4 & 52 & 4 & 53 & 4 & 54 & 4 & 55\end{array}$

$\begin{array}{llllllllll}4 & 56 & 4 & 57 & 4 & 91 & 5 & 16 & 5 & 17\end{array}$

$\begin{array}{llll}5 & 18 & 5 & 91\end{array}$

$\begin{array}{lllllllllll} & 1 & 452 & & & & & & & & \end{array}$

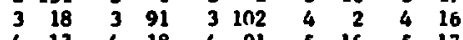

$33 \quad 81005 \quad 137 !$

$\begin{array}{llllllllll}4 & 17 & 4 & 18 & 4 & 91 & 5 & 16 & 5 & 17\end{array}$

$\begin{array}{lll}65 & 8007 & 3335\end{array}$

$\begin{array}{lllllllllll} & & 1 & 452 & & & & & & & \end{array}$

$\begin{array}{llllllllll}3 & 17 & 3 & 18 & 3 & 51 & 3 & 52 & 3 & 53 \\ 3 & 54 & 3 & 55 & 3 & 56 & 3 & 57 & 3 & 58\end{array}$

$\begin{array}{llllllllll}3 & 59 & 3 & 60 & 3 & 91 & 3 & 102 & 4 & 2\end{array}$

$\begin{array}{llllllllll}4 & 16 & 4 & 17 & 4 & 18 & 4 & 51 & 4 & 52\end{array}$

$\begin{array}{llllllllll}4 & 53 & 4 & 54 & 4 & 55 & 4 & 56 & 4 & 57 \\ 4 & 58 & 4 & 59 & 4 & 60 & 4 & 91 & 5 & 16\end{array}$

(cont inued) $\begin{array}{rllll}17 & 5 & 18 & 5 & 91\end{array}$

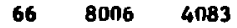

$87 \quad 8008 \quad 1876$

$401 \quad 8009 \quad 5819$ 
EHDR/A-801 (continued)

\begin{tabular}{|c|c|c|c|c|c|c|c|c|}
\hline \multirow{3}{*}{$\frac{\text { Isotop }}{\text { Pu-241 }}$} & \multicolumn{8}{|c|}{ React ton Typa } \\
\hline & 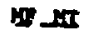 & \multicolumn{2}{|c|}{$\mathrm{Mr} \mathrm{HT}$} & \multicolumn{2}{|c|}{ IE Ir } & LIP & \multicolumn{2}{|c|}{ UP } \\
\hline & $\begin{array}{rr}2 & 151 \\
3 & 17 \\
4 & 16 \\
5 & 17\end{array}$ & $\begin{array}{l}3 \\
3 \\
4 \\
5\end{array}$ & $\begin{array}{l}1 \\
18 \\
17 \\
18\end{array}$ & $\begin{array}{l}3 \\
3 \\
4 \\
5\end{array}$ & $\begin{array}{l}2 \\
91 \\
18 \\
91\end{array}$ & $\begin{array}{rr}3 & 3 \\
3 & 102 \\
4 & 91\end{array}$ & $\begin{array}{l}3 \\
4 \\
5\end{array}$ & $\begin{array}{r}16 \\
2 \\
16\end{array}$ \\
\hline
\end{tabular}

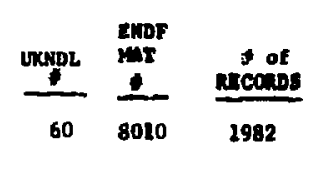

Ka-23 3102

$\begin{array}{lll}\mathrm{Mg}-24 & 3 \mathrm{LS}\end{array}$

$\begin{array}{lllll}A 1-27 & 3 & 1112 & 3 & 107\end{array}$

A1-27 3107

A]-27 3107

Si=28 3103

P-31 3103

S-32 3103

s-32 3103

S-34 3107

C1-35 $\quad 3107$

$\begin{array}{lllll}\text { Sc }-45 & 3 & 16 & 3 & 26\end{array}$

$224 \quad 6011 \quad 78$

Mn-55 3102

Fe-54 3103

Fe-54 3103

Fe-56 3103

Fe-56 3103

$\mathrm{Fe}-56 \quad 3 \quad 103$

Co-59 3102

N1-58 $3 \quad 16 \quad 3103$

$\begin{array}{lllll}\mathrm{Cu}-63 & 3 & 16 & 3 & 102\end{array}$

Cu-63 316

Cu-65 316

Y-89 $\quad 3 \quad 16$

$2 r-90 \quad 3 \quad 16$

Rh-103 316

$\begin{array}{lll}225 & 80 i 2 & 57\end{array}$

$\begin{array}{lll}226 & 8013 & 81\end{array}$

$95 \quad 8014 \quad 30$

$96 \quad 8015 \quad 39$

$\begin{array}{lll}227 & 8016 & 39\end{array}$

$\begin{array}{lll}228 & 8017 & 118\end{array}$

$229 \quad 8018 \quad 55$

$97 \quad 8019 \quad 41$

$230 \quad 8020 \quad 65$

$231 \quad 8021 \quad 29$

$207 \quad 8022 \quad 30$

$232 \quad 8023 \quad 202$

$63 \quad 8024 \quad 53$

$233 \quad 8025 \quad 37$

$62 \quad 8026 \quad 47$

$\begin{array}{lll}234 & 8027 & 34\end{array}$

$98 \quad 8028 \quad 30$

$\begin{array}{lll}235 & 8029 & 86\end{array}$

$236 \quad 8030 \quad 57$

$237 \quad 8031 \quad 89$

$\begin{array}{lll}99 & 8032 & 25\end{array}$

$100 \quad 8033 \quad 26$

$208 \quad 8034 \quad 22$

$238 \quad 8035 \quad 27$

$204 \quad 8036 \quad 27$

(cont Inued) 
ENDE/A-80I (continued)

\begin{tabular}{|c|c|c|c|c|c|c|}
\hline Isotope & & MrT & $\begin{array}{l}\text { Resction Typa" } \\
\text { UP MT }\end{array}$ & URIDL & $\begin{array}{c}\text { ENDF } \\
\mathbf{M T} \\
\bullet \\
\end{array}$ & $\begin{array}{c}\text { of } \\
\text { REconds } \\
\end{array}$ \\
\hline Rh-103 & $\mathbf{3}$ & 51 & 451 & .94 & 8037 & 59 \\
\hline In-I15 & 3 & 51 & & 239 & 8038 & 42 \\
\hline $1-127$ & 3 & 16 & & 240 & 8039 & 28 \\
\hline Gd & 3 & $\mathbf{I}$ & 3102 & 223 & 8040 & 40 \\
\hline$T m-169$ & 3 & 16 & & 209 & 8041 & 23 \\
\hline Lu- 175 & 3 & 26 & & 210 & 8042 & 23 \\
\hline \multirow[t]{2}{*}{ Th- 232} & 3 & 18 & 3102 & 242 & 8043 & 186 \\
\hline & & & & & & 24402 \\
\hline
\end{tabular}

*See ENDF-102 Vol 1 for reaction type values, 
OKHDL Data Library Recelved 5/5/71 Veraion 2

Reaction Type*

Isotope MF MT MR MT MR MT MF MT MF MT Pu-240

$\begin{array}{rrrrrrrrrr}2 & 151 & 3 & 1 & 3 & 2 & 3 & 3 & 3 & 16 \\ 3 & 17 & 3 & 18 & 3 & 51 & 3 & 52 & 3 & 53 \\ 3 & 91 & 3 & 102 & 4 & 2 & 4 & 16 & 4 & 17 \\ 4 & 18 & 4 & 51 & 4 & 52 & 4 & 53 & 4 & 91 \\ 5 & 16 & 5 & 17 & 5 & 18 & 5 & 91 & & \\ 1 & 452 & & & & & & & & \end{array}$

Th-232

$\begin{array}{rrrrrrrrrr}2 & 151 & 3 & 1 & 3 & 2 & 3 & 3 & 3 & 16 \\ 3 & 17 & 3 & 18 & 3 & 91 & 3 & 102 & 4 & 2 \\ 4 & 16 & 4 & 17 & 4 & 18 & 4 & 91 & 5 & 16 \\ 5 & 17 & 5 & 18 & 5 & 91 & & & & \\ 1 & 452 & & & & & & & & \end{array}$

$v-234$

$\begin{array}{llllllllll}2 & 151 & 3 & 1 & 3 & 2 & 3 & 3 & 3 & 16\end{array}$ $\begin{array}{rrrrrrrrrr}3 & 17 & 3 & 18 & 3 & 51 & 3 & 52 & 3 & 53 \\ 3 & 54 & 3 & 55 & 3 & 56 & 3 & 91 & 3 & 102\end{array}$ $\begin{array}{rrrrrrrrrr}3 & 54 & 3 & 55 & 3 & 56 & 3 & 91 & 3 & 102 \\ 4 & 2 & 4 & 16 & 4 & 17 & 4 & 18 & 4 & 51\end{array}$ $\begin{array}{lrllllllll}4 & 2 & 4 & 16 & 4 & 17 & 4 & 18 & 4 & 51 \\ 4 & 52 & 4 & 53 & 4 & 54 & 4 & 55 & 4 & 56\end{array}$ $\begin{array}{llllllllll}4 & 91 & 5 & 16 & 5 & 17 & 5 & 18 & 5 & 91\end{array}$ $\begin{array}{llllllllll}4 & 91 & 5 & 16 & 5 & 17 & 5 & 18 & 5 & 91\end{array}$

$U-236$

$\begin{array}{llllllllll}2 & 151 & 3 & 1 & 3 & 2 & 3 & 3 & 3 & 16\end{array}$

$\begin{array}{llllllllll}3 & 17 & 3 & 18 & 3 & 51 & 3 & 52 & 3 & 53\end{array}$

$\begin{array}{llllllllll}3 & 54 & 3 & 55 & 3 & 56 & 3 & 91 & 3 & 102\end{array}$

$\begin{array}{llllllllll}4 & 2 & 4 & 16 & 4 & 17 & 4 & 18 & 4 & 51\end{array}$

$\begin{array}{llllllllll}4 & 52 & 4 & 53 & 4 & 54 & 4 & 55 & 4 & 56\end{array}$

$\begin{array}{llllllllll}4 & 91 & 5 & 16 & 5 & 17 & 5 & 18 & 5 & 91\end{array}$

1452

$v-238$

$\begin{array}{llllllllll}2 & 151 & 3 & 1 & 3 & 2 & 3 & 3 & 3 & 16\end{array}$

$\begin{array}{llllllllll}3 & 17 & 3 & 18 & 3 & 51 & 3 & 52 & 3 & 53\end{array}$

$\begin{array}{llllllllll}3 & 54 & 3 & 55 & 3 & 56 & 3 & 57 & 3 & 58\end{array}$

$\begin{array}{llllllllll}3 & 59 & 3 & 60 & 3 & 91 & 3 & 102 & 4 & 2\end{array}$

$\begin{array}{lllllllrll}4 & 16 & 4 & 17 & 4 & 18 & 4 & 51 & 4 & 52\end{array}$

$\begin{array}{llllllllll}4 & 53 & 4 & 54 & 4 & 55 & 4 & 56 & 4 & 57\end{array}$

$\begin{array}{llllllllll}4 & 58 & 4 & 59 & 4 & 60 & 4 & 91 & 5 & 16\end{array}$

$\begin{array}{llllll}5 & 17 & 5 & 18 & 5 & 91\end{array}$

$\mathrm{Pu}-238$$$
2151
$$

$\begin{array}{llllll}2 & 151 & 3 & 1 & 3 & 2\end{array}$

$\begin{array}{llllll}3 & 17 & 3 & 18 & 3 & 91 \\ 4 & 16 & 4 & 17 & 4 & 18\end{array}$

$\begin{array}{rrrrrr}5 & 17 & 5 & 18 & 5 & 91\end{array}$

1452

Pa-233

2151

$\begin{array}{llll}3 & 1 & 3 & 2\end{array}$

$\begin{array}{ll}4 & 18 \\ 1 & 45\end{array}$

1452

Np $-237 \quad 3 \quad 18$ $\underbrace{\text { UKtDL }}_{77} \frac{\begin{array}{l}\text { ENDF } \\ \text { M044 }\end{array}}{1180}$

$22 \quad 8045 \quad 1045$

$74 \quad 8046 \quad 1170$

$\begin{array}{lll}75 & 8047 & 1025\end{array}$

$\begin{array}{lll}76 & 8048 & 1069\end{array}$

$216 \quad 8049 \quad 620$

$\begin{array}{rrrr}3 & 3 & 3 & 16 \\ 3 & 102 & 4 & 2\end{array}$

$\begin{array}{rrrr}4 & 91 & 5 & 16\end{array}$

$86 \quad 8050 \quad 981$

$61 \quad 8051 \quad 86$

(continued) 
ENDF/A-802 (continued)

Reaction Type*

Isotope MF MT MF MT MF MT WTE MTI MFT

$\mathrm{Na}$

$\begin{array}{rrrrrrrrrr}2 & 151 & 3 & 1 & 3 & 2 & 3 & 3 & 3 & 16 \\ 3 & 51 & 3 & 52 & 3 & 53 & 3 & 54 & 3 & 55 \\ 3 & 56 & 3 & 57 & 3 & 91 & 3 & 102 & 3 & 103 \\ 3 & 107 & 4 & 2 & 4 & 16 & 4 & 51 & 4 & 52 \\ 4 & 53 & 4 & 54 & 4 & 55 & 4 & 56 & 4 & 57 \\ 4 & 91 & 5 & 16 & 5 & 91 & & & & \end{array}$

K

$\begin{array}{rrrrrrrrrr}2 & 151 & 3 & 1 & 3 & 2 & 3 & 3 & 3 & 4 \\ 3 & 16 & 3 & 22 & 3 & 51 & 3 & 52 & 3 & 53 \\ 3 & 54 & 3 & 55 & 3 & 56 & 3 & 91 & 3 & 102 \\ 3 & 103 & 3 & 107 & 4 & 2 & 4 & 16 & 4 & 22 \\ 4 & 51 & 4 & 52 & 4 & 53 & 4 & 54 & 4 & 55 \\ 4 & 56 & 4 & 91 & 5 & 16 & 5 & 22 & 5 & 91\end{array}$

84

8053

2804

A1

$\begin{array}{rrrrrrrrrr}2 & 151 & 3 & 1 & 3 & 2 & 3 & 16 & 3 & 51 \\ 3 & 52 & 3 & 53 & 3 & 54 & 3 & 55 & 3 & 56 \\ 3 & 57 & 3 & 58 & 3 & 59 & 3 & 91 & 3 & 102 \\ 3 & 113 & 3 & 107 & 4 & 2 & 4 & 16 & 4 & 51 \\ 4 & 52 & 4 & 53 & 4 & 54 & 4 & 55 & 4 & 56 \\ 4 & 57 & 4 & 58 & 4 & 59 & 4 & 91 & 5 & 16 \\ 5 & 91 & & & & & & & & \end{array}$

$2 \mathbf{r}$

$\begin{array}{rrrrrrrrrr}2 & 151 & 3 & 1 & 3 & 2 & 3 & 3 & 3 & 16 \\ 3 & 51 & 3 & 52 & 3 & 53 & 3 & 54 & 3 & 55 \\ 3 & 56 & 3 & 57 & 3 & 58 & 3 & 91 & 3 & 102 \\ 3 & 103 & 4 & 2 & 4 & 16 & 4 & 51 & 4 & 52 \\ 4 & 53 & 4 & 54 & 4 & 55 & 4 & 56 & 4 & 57\end{array}$

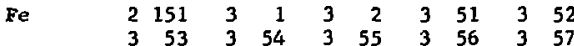

$\begin{array}{llllllll}4 & 58 & 4 & 91 & 5 & 16 & 5 & 91\end{array}$

$\begin{array}{llllllllll}3 & 58 & 3 & 59 & 3 & 60 & 3 & 91 & 3 & 102\end{array}$

$\begin{array}{llllllllll}3 & 103 & 3 & 107 & 4 & 2 & 4 & 51 & 4 & 52\end{array}$

$\begin{array}{llllllllll}4 & 53 & 4 & 54 & 4 & 55 & 4 & 56 & 4 & 57 \\ 4 & 58 & 4 & 59 & 4 & 60 & 4 & 91 & 5 & 91\end{array}$

Cu

$$
\begin{array}{rrrrrrrrrr}
2 & 151 & 3 & 1 & 3 & 2 & 3 & 4 & 3 & 16 \\
3 & 51 & 3 & 52 & 3 & 53 & 3 & 54 & 3 & 55 \\
3 & 56 & 3 & 57 & 3 & 58 & 3 & 59 & 3 & 91 \\
3 & 102 & 3 & 103 & 3 & 107 & 4 & 2 & 4 & 16 \\
4 & 51 & 4 & 52 & 4 & 53 & 4 & 54 & 4 & 55 \\
4 & 56 & 4 & 57 & 4 & 58 & 4 & 59 & 4 & 91 \\
5 & 16 & 5 & 91 & & & & & &
\end{array}
$$

Mo

$\begin{array}{rrrrrrrrrrrrr}2 & 151 & 3 & 1 & 3 & 2 & 3 & 3 & 3 & 4 & 81 & 8058 & 1457 \\ 3 & 16 & 3 & 17 & 3 & 22 & 3 & 51 & 3 & 52 & & & \\ 3 & 53 & 3 & 54 & 3 & 91 & 3 & 102 & 3 & 103 & & & \\ 4 & 2 & 4 & 16 & 4 & 17 & 4 & 22 & 4 & 51 & & & \\ 4 & 52 & 4 & 53 & 4 & 54 & 4 & 91 & 5 & 16 & & & \\ 5 & 17 & 5 & 22 & 5 & 91 & & & & & & & \end{array}$

$35 \quad 8054 \quad 1306$

$82 \quad 8055 \quad 1958$

$91 \quad 8056 \quad 2938$

$\begin{array}{lll}73 & 8057 & 3274\end{array}$

$\begin{array}{llllllllll}3 & 16 & 3 & 17 & 3 & 22 & 3 & 51 & 3 & 52\end{array}$

$\begin{array}{llllllllll}3 & 53 & 3 & 54 & 3 & 91 & 3 & 102 & 3 & 103\end{array}$

$\begin{array}{llllll}5 & 17 & 5 & 22 & 5 & 91\end{array}$

(cont tnued) 
ENDF/A-802 (continued)

Reaction Type*

Isotope MP MT MF MT MP MT MP MT MFMT

$$
\begin{array}{rrrrrrrrrr}
2 & 151 & 3 & 1 & 3 & 2 & 3 & 3 & 3 & 16 \\
3 & 91 & 3 & 102 & 4 & 2 & 4 & 16 & 4 & 91
\end{array}
$$$$
\begin{array}{llll}
5 & 16 & 5 & 91
\end{array}
$$

Cr

$$
\begin{array}{rrrrrrrrrr}
2 & 151 & 3 & 1 & 3 & 2 & 3 & 3 & 3 & 16 \\
3 & 51 & 3 & 52 & 3 & 53 & 3 & 54 & 3 & 55 \\
3 & 56 & 3 & 57 & 3 & 58 & 3 & 91 & 3 & 102 \\
3 & 103 & 4 & 2 & 4 & 16 & 4 & 51 & 4 & 52 \\
4 & 53 & 4 & 54 & 4 & 55 & 4 & 56 & 4 & 57
\end{array}
$$

$\mathrm{Ni}$$$
\begin{array}{llllllllll}
2 & 15 & 3 & 1 & 3 & 2 & 3 & 3 & 3 & 16
\end{array}
$$$$
\begin{array}{llllllllll}
3 & 51 & 3 & 52 & 3 & 2 & 3 & 3 & 3 & 16 \\
3 & 56 & 3 & 57 & 3 & 58 & 3 & 54 & 3 & 55
\end{array}
$$$$
\begin{array}{llllllllll}
3 & 56 & 3 & 57 & 3 & 58 & 3 & 91 & 3 & 102
\end{array}
$$$$
\begin{array}{llllllllll}
3 & 103 & 3 & 107 & 4 & 2 & 4 & 16 & 4 & 51
\end{array}
$$$$
\begin{array}{llllllllll}
4 & 52 & 4 & 53 & 4 & 54 & 4 & 55 & 4 & 56
\end{array}
$$

$45 \quad 8060 \quad 2085$

46

8061

1460

ऋSee ENDF-102 vol 1 for reaction type values. 
ENDE/A-803

UKNDL, Data LIbrary Rece ived 5/5/71

Version 2

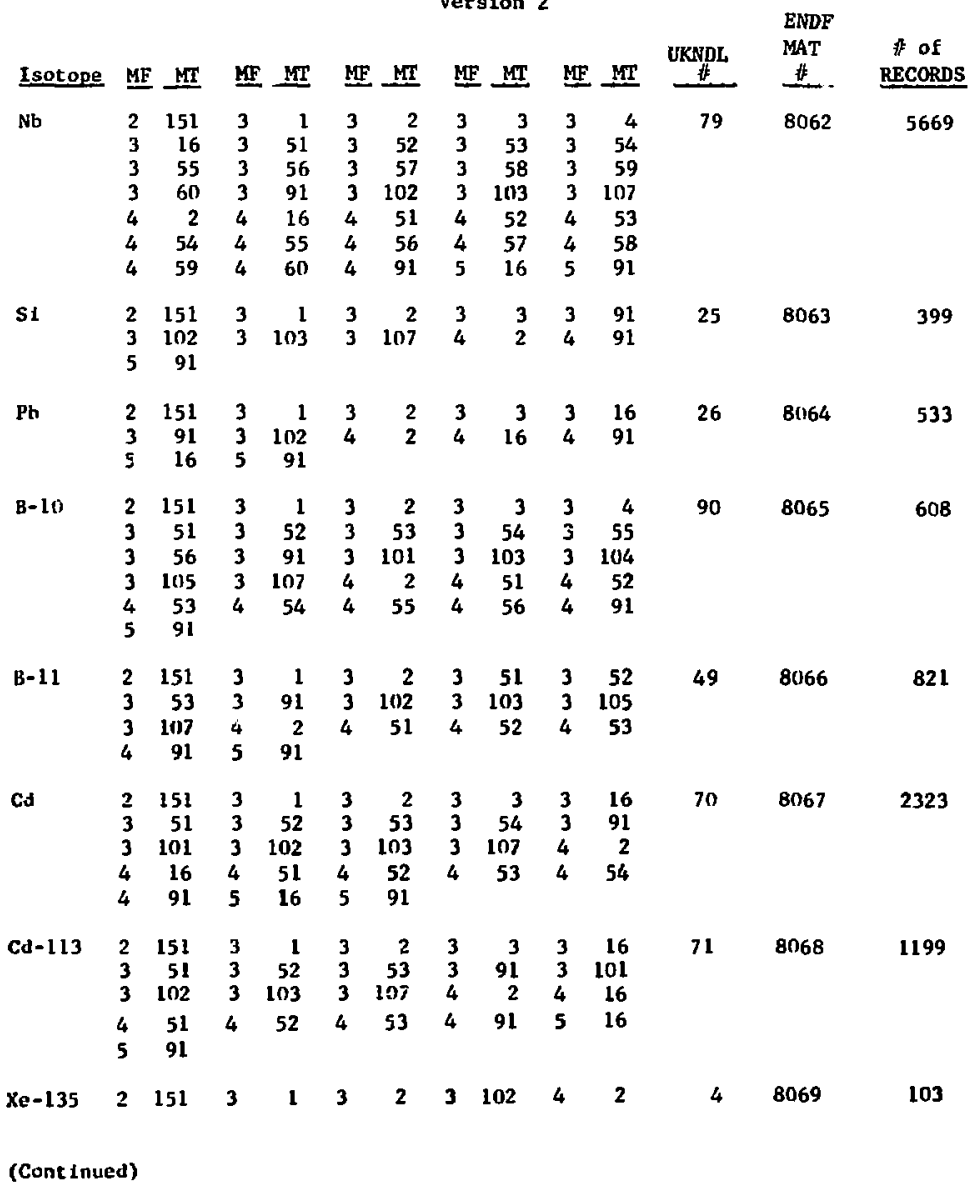


ENDF/A-8C,3 (continued)

\begin{tabular}{|c|c|c|c|c|c|c|c|c|c|c|c|c|c|}
\hline \multirow[b]{2}{*}{ Isotope } & \multicolumn{10}{|c|}{ Reaction Type * } & \multirow{2}{*}{$\begin{array}{c}\text { UKNDL } \\
\text { 将 }\end{array}$} & \multirow{2}{*}{$\begin{array}{l}\text { ENDE } \\
\text { MAT } \\
\sharp \#\end{array}$} & \multirow{2}{*}{$\begin{array}{c}\# \text { of } \\
\text { RECORDS }\end{array}$} \\
\hline & $\underline{\mathrm{MF}}$ & MT & $\underline{\mathrm{MF}}$ & $\underline{M r}$ & $\underline{M F}$ & MT & $\underline{M F}$ & MT & $\underline{\mathrm{MF}}$ & MT & & & \\
\hline$R-3\langle T\rangle$ & $\begin{array}{l}2 \\
4\end{array}$ & $\begin{array}{r}151 \\
2\end{array}$ & $\begin{array}{l}3 \\
4\end{array}$ & $\begin{array}{r}1 \\
16\end{array}$ & $\begin{array}{l}3 \\
5\end{array}$ & $\begin{array}{r}2 \\
16\end{array}$ & 3 & 3 & 3 & 16 & 252 & 80170 & 676 \\
\hline $\mathrm{He}-3$ & $\begin{array}{l}2 \\
3\end{array}$ & $\begin{array}{l}151 \\
104\end{array}$ & $\begin{array}{l}3 \\
4\end{array}$ & $\begin{array}{l}1 \\
2\end{array}$ & 3 & 2 & 3 & 3 & 3 & 103 & 220 & 8071 & 243 \\
\hline $\mathrm{He}-4$ & 2 & 151 & 3 & 1 & 3 & 2 & 4 & 2 & & & 221 & 8072 & 290 \\
\hline $\mathbf{L} \mathbf{1}-6$ & $\begin{array}{l}2 \\
3 \\
4 \\
5\end{array}$ & $\begin{array}{r}151 \\
24 \\
2 \\
24\end{array}$ & $\begin{array}{l}3 \\
3 \\
4\end{array}$ & $\begin{array}{r}1 \\
51 \\
22\end{array}$ & $\begin{array}{l}3 \\
3 \\
4\end{array}$ & $\begin{array}{r}2 \\
102 \\
24\end{array}$ & $\begin{array}{l}3 \\
3 \\
4\end{array}$ & $\begin{array}{r}3 \\
103 \\
51\end{array}$ & $\begin{array}{l}3 \\
3 \\
5\end{array}$ & $\begin{array}{r}22 \\
107 \\
22\end{array}$ & 214 & 8073 & 962 \\
\hline $\mathbf{L} 1-7$ & $\begin{array}{l}2 \\
3 \\
4 \\
5\end{array}$ & $\begin{array}{r}151 \\
22 \\
2 \\
16\end{array}$ & $\begin{array}{l}3 \\
3 \\
4 \\
5\end{array}$ & $\begin{array}{r}1 \\
24 \\
16 \\
22\end{array}$ & $\begin{array}{l}3 \\
3 \\
4 \\
5\end{array}$ & $\begin{array}{r}2 \\
51 \\
22 \\
24\end{array}$ & $\begin{array}{l}3 \\
3 \\
4\end{array}$ & $\begin{array}{r}3 \\
102 \\
24\end{array}$ & $\begin{array}{l}3 \\
3 \\
4\end{array}$ & $\begin{array}{r}16 \\
104 \\
51\end{array}$ & 215 & 8074 & 902 \\
\hline B & $\begin{array}{l}2 \\
3\end{array}$ & $\begin{array}{l}151 \\
101\end{array}$ & $\begin{array}{l}3 \\
4\end{array}$ & $\begin{array}{l}1 \\
2\end{array}$ & $\begin{array}{l}3 \\
4\end{array}$ & $\begin{array}{r}2 \\
91\end{array}$ & $\begin{array}{l}3 \\
5\end{array}$ & $\begin{array}{r}3 \\
91\end{array}$ & 3 & 91 & 57 & 8075 & 367 \\
\hline $\mathbf{N}$ & $\begin{array}{l}2 \\
3 \\
3 \\
4 \\
5\end{array}$ & $\begin{array}{r}151 \\
51 \\
104 \\
16 \\
91\end{array}$ & $\begin{array}{l}3 \\
3 \\
3 \\
4\end{array}$ & $\begin{array}{r}1 \\
52 \\
105 \\
51\end{array}$ & $\begin{array}{l}3 \\
3 \\
3 \\
4\end{array}$ & $\begin{array}{r}2 \\
91 \\
107 \\
52\end{array}$ & $\begin{array}{l}3 \\
3 \\
3 \\
4\end{array}$ & $\begin{array}{r}3 \\
102 \\
108 \\
91\end{array}$ & $\begin{array}{l}3 \\
3 \\
4 \\
5\end{array}$ & $\begin{array}{r}16 \\
103 \\
2 \\
16\end{array}$ & 259 & 8076 & 3664 \\
\hline $\mathbf{F}$ & $\begin{array}{l}2 \\
3 \\
5\end{array}$ & $\begin{array}{r}151 \\
91 \\
16\end{array}$ & $\begin{array}{l}3 \\
3 \\
5\end{array}$ & $\begin{array}{r}1 \\
101 \\
91\end{array}$ & $\begin{array}{l}3 \\
4\end{array}$ & $\begin{array}{l}2 \\
2\end{array}$ & $\begin{array}{l}3 \\
4\end{array}$ & $\begin{array}{r}3 \\
16\end{array}$ & $\begin{array}{l}3 \\
4\end{array}$ & $\begin{array}{l}16 \\
91\end{array}$ & 23 & 8077 & 398 \\
\hline Gl & $\begin{array}{l}2 \\
3 \\
4\end{array}$ & $\begin{array}{r}151 \\
91 \\
16\end{array}$ & $\begin{array}{l}3 \\
3 \\
4\end{array}$ & $\begin{array}{r}1 \\
102 \\
91\end{array}$ & $\begin{array}{l}3 \\
3 \\
5\end{array}$ & $\begin{array}{r}2 \\
103 \\
16\end{array}$ & $\begin{array}{l}3 \\
3 \\
5\end{array}$ & $\begin{array}{r}3 \\
107 \\
19\end{array}$ & $\begin{array}{l}3 \\
4\end{array}$ & $\begin{array}{r}16 \\
2\end{array}$ & 141 & 8078 & 563 \\
\hline $\mathrm{Ca}$ & $\begin{array}{l}2 \\
3 \\
5\end{array}$ & $\begin{array}{r}151 \\
102 \\
91\end{array}$ & $\begin{array}{l}3 \\
3\end{array}$ & $\begin{array}{r}1 \\
103\end{array}$ & $\begin{array}{l}3 \\
3\end{array}$ & $\begin{array}{r}2 \\
107\end{array}$ & $\begin{array}{l}3 \\
4\end{array}$ & $\begin{array}{l}3 \\
2\end{array}$ & $\begin{array}{l}3 \\
4\end{array}$ & $\begin{array}{l}91 \\
91\end{array}$ & 138 & 8079 & 362 \\
\hline $\mathrm{ri}$ & $\begin{array}{l}2 \\
3 \\
3 \\
4 \\
5\end{array}$ & $\begin{array}{r}151 \\
51 \\
102 \\
51 \\
16\end{array}$ & $\begin{array}{l}3 \\
3 \\
3 \\
4 \\
5\end{array}$ & $\begin{array}{r}1 \\
52 \\
103 \\
52 \\
91\end{array}$ & $\begin{array}{l}3 \\
3 \\
3 \\
4\end{array}$ & $\begin{array}{r}2 \\
53 \\
107 \\
53\end{array}$ & $\begin{array}{l}3 \\
3 \\
4 \\
4\end{array}$ & $\begin{array}{r}3 \\
54 \\
2 \\
54\end{array}$ & $\begin{array}{l}3 \\
3 \\
4 \\
4\end{array}$ & $\begin{array}{l}16 \\
91 \\
16 \\
91\end{array}$ & 190 & 8080 & 1150 \\
\hline
\end{tabular}

(cont inued) 
ENDF/A-803 (continued)

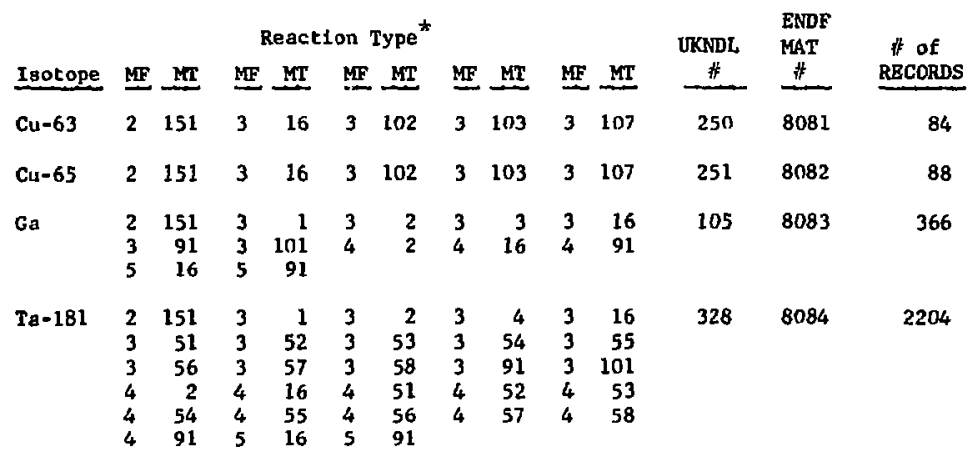

*See ENDF-102 vol 1 for reaction type values. 
ENDF/A-804

UKNDL Data Library Received 5/5/71

\begin{tabular}{|c|c|c|c|c|c|c|c|c|c|c|c|c|c|}
\hline \multirow[b]{2}{*}{ Isotope } & \multicolumn{10}{|c|}{ Reaction Type* } & \multirow{2}{*}{\multicolumn{2}{|c|}{$\begin{array}{c}\text { ENDF } \\
\text { MAT } \\
\#\end{array}$}} & \multirow{2}{*}{$\begin{array}{c}\text { f of } \\
\text { RECORDS }\end{array}$} \\
\hline & $M F$ & $M T$ & $M F$ & MT & $\mathbf{M F}$ & MT & MF & $\mathbf{M I I}$ & $M F$ & MT & & & \\
\hline$u=197$ & $\begin{array}{l}2 \\
3 \\
3 \\
3 \\
4 \\
4 \\
4\end{array}$ & $\begin{array}{r}151 \\
26 \\
55 \\
91 \\
26 \\
55 \\
91\end{array}$ & $\begin{array}{l}3 \\
3 \\
3 \\
3 \\
4 \\
4 \\
5\end{array}$ & $\begin{array}{r}1 \\
51 \\
56 \\
102 \\
51 \\
56 \\
16\end{array}$ & $\begin{array}{l}3 \\
3 \\
3 \\
3 \\
4 \\
4 \\
5\end{array}$ & $\begin{array}{r}2 \\
52 \\
57 \\
103 \\
52 \\
57 \\
26\end{array}$ & $\begin{array}{l}3 \\
3 \\
3 \\
4 \\
4 \\
4 \\
5\end{array}$ & $\begin{array}{r}3 \\
53 \\
58 \\
2 \\
53 \\
58 \\
91\end{array}$ & $\begin{array}{l}3 \\
3 \\
3 \\
4 \\
4 \\
4\end{array}$ & $\begin{array}{l}16 \\
54 \\
59 \\
16 \\
54 \\
59\end{array}$ & 222 & 8085 & 968 \\
\hline
\end{tabular}

Au-197 $3 \quad 102$

$\begin{array}{llllllllllll}\text { Fp } & 3 & 1 & 3 & 2 & 3 & 3 & 3 & 16 & 3 & 91 & 106\end{array}$ $\begin{array}{rrrrrrrrrr}3 & 101 & 4 & 2 & 4 & 16 & 4 & 91 & 5 & 16\end{array}$

$\begin{array}{lllllll}\text { VAC } & 3 & 1 & 3 & 2 & 4 & 2\end{array}$

$\begin{array}{lllllllll}1 / V & 3 & 1 & 3 & 2 & 3 & 102 & 4 & 2\end{array}$

$\begin{array}{lllllll}\text { SGAT } & 3 & 1 & 3 & 2 & 4 & 2\end{array}$

$\begin{array}{lllllllll}\text { ABS } & 3 & 1 & 3 & 2 & 3 & 102 & 4 & 2\end{array}$
$241 \quad 8086 \quad 111$

$8087 \quad 350$

$172 \quad 8088 \quad 39$

$55 \quad 8089 \quad 45$

$56 \quad 8090 \quad 39$

151

809145

*See ENDF-102 vo1 1 for reaction type values. 
U.K. LIBRARY RECEIVED JULY 2, 1973

Version 3

Nucleus uK Librexy Ho, No, of Cerde Tape No.

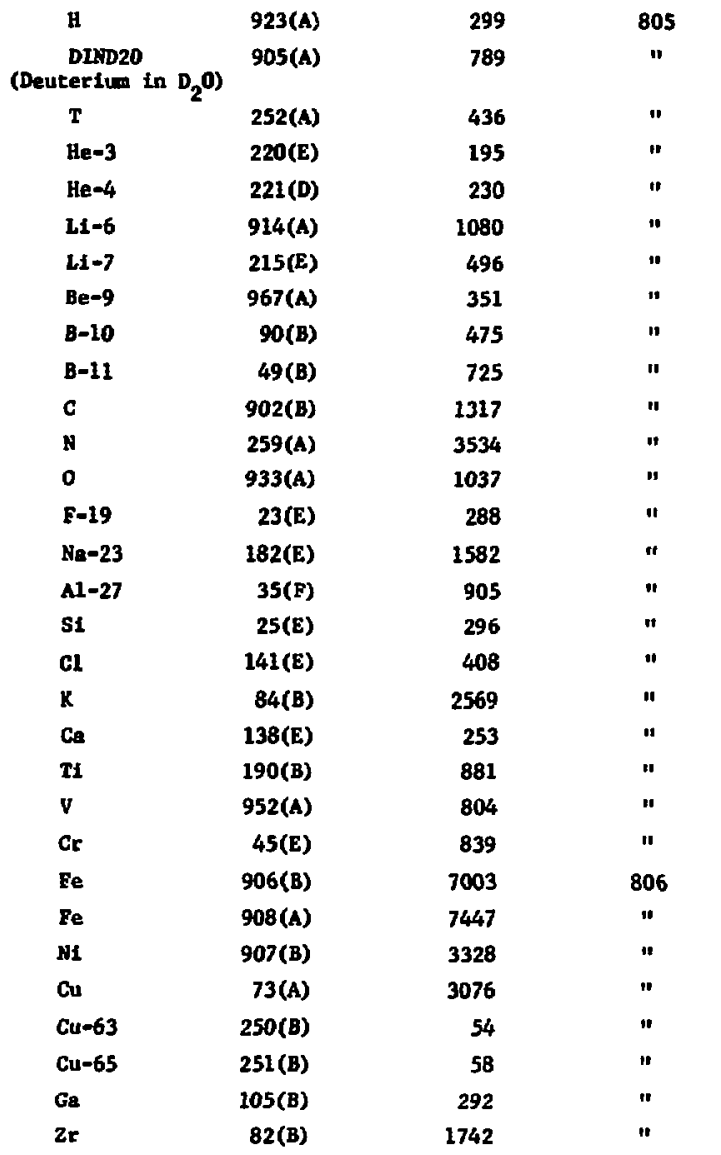


U.K. Library (continued)

\begin{tabular}{|c|c|c|c|}
\hline Nucleus & UK Library lo, & No, of Cards: & Inpe No. \\
\hline $\mathbf{N b}-93$ & $79(c)$ & 5579 & 807 \\
\hline Ho & 81 (B) & 1309 & $"$ \\
\hline$A g=107$ & $973(A)$ & 1107 & $"$ \\
\hline$A g-109$ & $974(A)$ & 1205 & $"$ \\
\hline cd & $70(A)$ & 2203 & $"$ \\
\hline$c d-113$ & $71(B)$ & 1088 & $"$ \\
\hline Xe-135 & $4(F)$ & 66 & " \\
\hline Eu-151 & $921(A)$ & 3458 & 1 \\
\hline Eu-153 & $922(A)$ & 4055 & $"$ \\
\hline $\mathbf{T a}$ & 328 (B) & 2069 & $"$ \\
\hline H & $213(B)$ & 238 & $"$ \\
\hline$A u-197$ & 222 (E) & 690 & 804 \\
\hline Pb & $26(C)$ & 545 & $"$ \\
\hline Th-232 & 930 & 774 & 11 \\
\hline$P a-233$ & $86(A)$ & 900 & $"$ \\
\hline$v-233$ & $87(B)$ & 1835 & " \\
\hline $\mathrm{U}-234$ & $953(A)$ & 823 & $"$ \\
\hline $\mathrm{U}-235$ & 159 (B) & 4354 & $"$ \\
\hline $\mathrm{U}-236$ & $954(A)$ & 749 & $"$ \\
\hline $\mathrm{U}-238$ & $160(A)$ & 5886 & $1 "$ \\
\hline $\mathrm{U}-239$ & $276(A)$ & 1119 & $"$ \\
\hline$U-240$ & 277 (A) & 1060 & " \\
\hline$P u-238$ & 274 (A) & 438 & " \\
\hline $\mathrm{Pu}=239$ & $161(A)$ & 3334 & 809 \\
\hline Pu-239 & $404(B)$ & 4382 & $"$ \\
\hline Pu-240 & $402(B)$ & 2669 & $"$ \\
\hline $\mathrm{Pu}-240$ & 77 (B) & 1052 & $n$ \\
\hline$P u=241$ & $403(B)$ & 1661 & 11 \\
\hline Pu-241 & $60(A)$ & 2155 & $"$ \\
\hline Pu-242 & $975(A)$ & 1069 & $"$ \\
\hline Am-241 & $956(\Lambda)$ & 1213 & 11 \\
\hline$A m=243$ & $957(A)$ & 574 & 11 \\
\hline Cm-244 & $976(A)$ & 1649 & $"$ \\
\hline
\end{tabular}


The contents of the Speng Library are as follows:

$\begin{array}{lll}\text { Pu-239 } & \text { Na-23 } & \text { Pu-240 } \\ \text { B-10 } & \text { D-2 } & \text { Al } \\ \text { B-11 } & F & \text { Mr } \\ \text { St } & \text { Zr } & \text { Ni } \\ \text { He } & \text { Mo } & \text { Cr } \\ \text { Li-6 } & \text { (Fiss. P. 239) } & \text { U-235 } \\ \text { Li-7 } & \text { W-186 } & \text { Cu } \\ \text { Ta } & \text { Au-197 } & \text { H-1 } \\ \text { Er } & \text { Fe } & \text { Pu-241 } \\ \text { U-238 } & \text { B } & \end{array}$


LLL. ENDL Data In ENDE/B Format

Recelved Jan. 25, 1974

\begin{tabular}{|c|c|c|}
\hline Macerial & MAT Number & $\underline{N}, x y$ Included \\
\hline Neutron & 7000 & $-\infty$ \\
\hline Hydrogen & 7001 & Yes \\
\hline Deuteron & $7002^{\star}$ & Yes \\
\hline Triton & 7003 & $-\ldots$ \\
\hline $\mathrm{He}^{3}$ & 7004 & Yes \\
\hline $\mathrm{He}^{4}$ & 7005 & --- \\
\hline $\mathrm{Li}^{6}$ & 7006 & Yes \\
\hline . & 7007 & Yes \\
\hline & 7008 & Yes \\
\hline 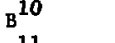 & 7009 & Yes \\
\hline & 7010 & Yes \\
\hline & 7011 & Yes \\
\hline$N^{14}$ & 7012 & Yes \\
\hline $0^{16}$ & 7013 & Yes \\
\hline$F^{19}$ & 7014 & Yes \\
\hline $\mathrm{Na}^{23}$ & 7015 & Yes \\
\hline & 7016 & Yes \\
\hline $\mathrm{Al}^{21}$ & 7017 & Yes \\
\hline & 7018 & Yes \\
\hline$P_{32}^{31}$ & 7019 & Yes \\
\hline & 7020 & Yes \\
\hline $\mathrm{Cl}^{\text {Nat }}$ & 7021 & Yes \\
\hline$A x^{\operatorname{Nat}}$ & 7022 & Yes \\
\hline
\end{tabular}

*The energy-angle distribution of secondary neutrons from the $n, 2 n$ reaction for $D$ is represented by an energy-angle Legendre expansion in the ENDL system. No equivalent representation exists in the ENDF/B system. Consequently, the representation in the translated form is deficlent. 
LLL ENDL Daca in ENDF/B Pormat

\begin{tabular}{|c|c|c|}
\hline Material & MAT Numer & $\underline{N}, x Y$ Included \\
\hline$k^{\text {Nat }}$ & 7023 & Yes \\
\hline & 7024 & Yes \\
\hline & 7025 & Yes \\
\hline & 7026 & No (Planned) \\
\hline & 7027 & No (Planned) \\
\hline & 7028 & Yes \\
\hline & 7029 & Yes \\
\hline & 7030 & Yes \\
\hline & 7031 & Yes \\
\hline & 7032 & Yen \\
\hline & 7033 & No (Planned) \\
\hline & 7034 & Yes \\
\hline & 7035 & Yes \\
\hline & 7036 & No (P1anned) \\
\hline & 7037 & No (Planned) \\
\hline$C d^{\text {Nat }}$ & 7038 & Yes \\
\hline $\mathrm{Sn}^{\operatorname{Mac}}$ & 7039 & Yes \\
\hline $\mathrm{Ba}^{\mathrm{Mat}}$ & 7040 & Yes \\
\hline & 7041 & Yes \\
\hline & 7042 & Yes \\
\hline $\mathrm{Ho}_{181}^{203}$ & 7043 & Yes \\
\hline & 7044 & Yes \\
\hline$w$ & 7045 & Yes \\
\hline$P t_{197}^{\operatorname{Mat}}$ & 7046 & Yes \\
\hline & 7047 & Yes \\
\hline $\mathrm{Pb}_{232}^{\mathrm{Mat}}$ & 7048 & Yes \\
\hline $\begin{array}{l}\mathrm{Th}^{201} \\
233\end{array}$ & 7049 & Yes \\
\hline & 7050 & Yes \\
\hline
\end{tabular}


LLI ENDI, Data in ENDF/B Format

\begin{tabular}{|c|c|c|}
\hline Material & MAT Number & N, $x Y$ Included \\
\hline $\mathrm{u}^{234}$ & 7051 & Yes \\
\hline$u^{235}$ & 7052 & Yes \\
\hline $\mathrm{u}^{236}$ & 7053 & Yes \\
\hline$v^{237}$ & 7054 & Yes \\
\hline$u^{238}$ & 7055 & Yes \\
\hline $\mathrm{U}^{239}$ & 7056 & Yes \\
\hline $\mathrm{U}^{240}$ & 7057 & Yes \\
\hline $\mathrm{Np}^{237}$ & 7058 & Yes \\
\hline $\mathrm{Pu}^{238}$ & 7059 & Yes \\
\hline $\mathrm{Pu}^{239}$ & 7060 & Yes \\
\hline $\mathrm{Pu}^{240}$ & 7061 & Yes \\
\hline $\mathrm{Pu}^{241}$ & 7062 & Yes \\
\hline $\mathrm{Am}^{242}$ & 7063 & Yes \\
\hline$S c^{45}$ (Partial) & 7064 & No \\
\hline $\mathrm{Fe}^{54}$ (Partial) & 7065 & No \\
\hline $\mathrm{Fe}^{56}$ (Partial) & 7066 & No \\
\hline $\mathrm{Ie}^{58}$ (Partial) & 7067 & No \\
\hline $\operatorname{Re}^{185}($ Partial) & 7068 & No \\
\hline $\operatorname{Re}^{187}$ (Partial) & 7069 & No \\
\hline $\operatorname{Ir}{ }^{191}$ (Partial) & 7070 & No \\
\hline Ir $^{193}$ (Parttal) & 7071 & No \\
\hline Fisston Product & 7072 & No \\
\hline
\end{tabular}




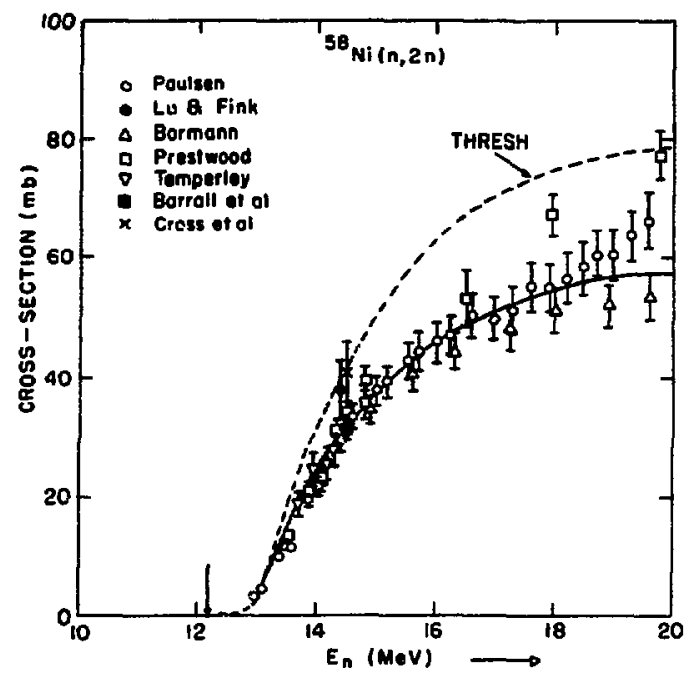

P1. 1. ${ }^{58}$ NI $(n, 2 n)$ Cross-Section 
APPENDLX 0

Requests for CTR Related Projects

$-47-$ 


\begin{tabular}{|c|c|c|c|c|c|c|c|}
\hline Nucleus & $\begin{array}{c}\text { Cook } \\
\text { Library }\end{array}$ & $\begin{array}{l}\text { Livola1 } \\
\text { "1500" } \\
\text { Serie日 }\end{array}$ & UKNDL & Speng & $\begin{array}{c}\text { ENDF/B } \\
\text { III } \\
\text { MAT nO } \\
\end{array}$ & $\begin{array}{l}\text { Any } \\
\text { Other }\end{array}$ & $\begin{array}{l}\text { THRESH } \\
\text { Calculation }\end{array}$ \\
\hline H & & & & & 1148 & & \\
\hline He & & & & & $108 B$ & & \\
\hline${ }^{3} \mathrm{He}$ & & & 8071 & & 1146 & & \\
\hline${ }^{6},{ }^{7} \mathrm{LI}$ & & & & & $\begin{array}{l}1115 \\
1116\end{array}$ & & \\
\hline${ }^{9} \mathrm{Be}$ & & & & & 1154 & & \\
\hline${ }^{10} \mathrm{~B}$ & & & 8065 & & 1155 & & \\
\hline${ }^{31} \mathrm{~B}$ & & & & & 1160 & & \\
\hline${ }^{23} \mathrm{C}$ & & & & & 1165 & & \\
\hline $\mathbf{N}$ & & & & & 1133 & & \\
\hline 0 & & & & & 1134 & & \\
\hline $\mathbf{F}$ & & & 8077 & & & & \\
\hline${ }^{\mathrm{a}} \mathrm{Na}$ & & & & & 1156 & & \\
\hline $\mathrm{MB}$ & & & & & 1014 & & \\
\hline Al & & & & & 1135 & & \\
\hline${ }^{a \theta} \mathrm{SI}$ & & & 8016 & & & & \\
\hline \multicolumn{8}{|l|}{${ }^{2 \theta} \mathrm{S} 1$} \\
\hline \multicolumn{8}{|l|}{${ }^{30} \mathrm{~S} 1$} \\
\hline$P$ & & & 8017 & & & & \\
\hline${ }^{39_{\mathrm{K}}}$ & & & & & 1150 & & \\
\hline \multicolumn{8}{|l|}{${ }^{41} \mathrm{~K}$} \\
\hline${ }^{45} \mathrm{Sc}$ & & & & & & & $x$ \\
\hline${ }^{46} \mathrm{~T} 1$ & & & & & & & $x$ \\
\hline${ }^{47} \mathrm{TI}$ & & & & & & & $x$ \\
\hline${ }^{8 \theta} \mathrm{TI}$ & & & & & & & $x$ \\
\hline${ }^{40} \mathrm{Ti}$ & & & & & & & $x$ \\
\hline${ }^{E} O_{T i}$ & & & & & & & $x$ \\
\hline
\end{tabular}




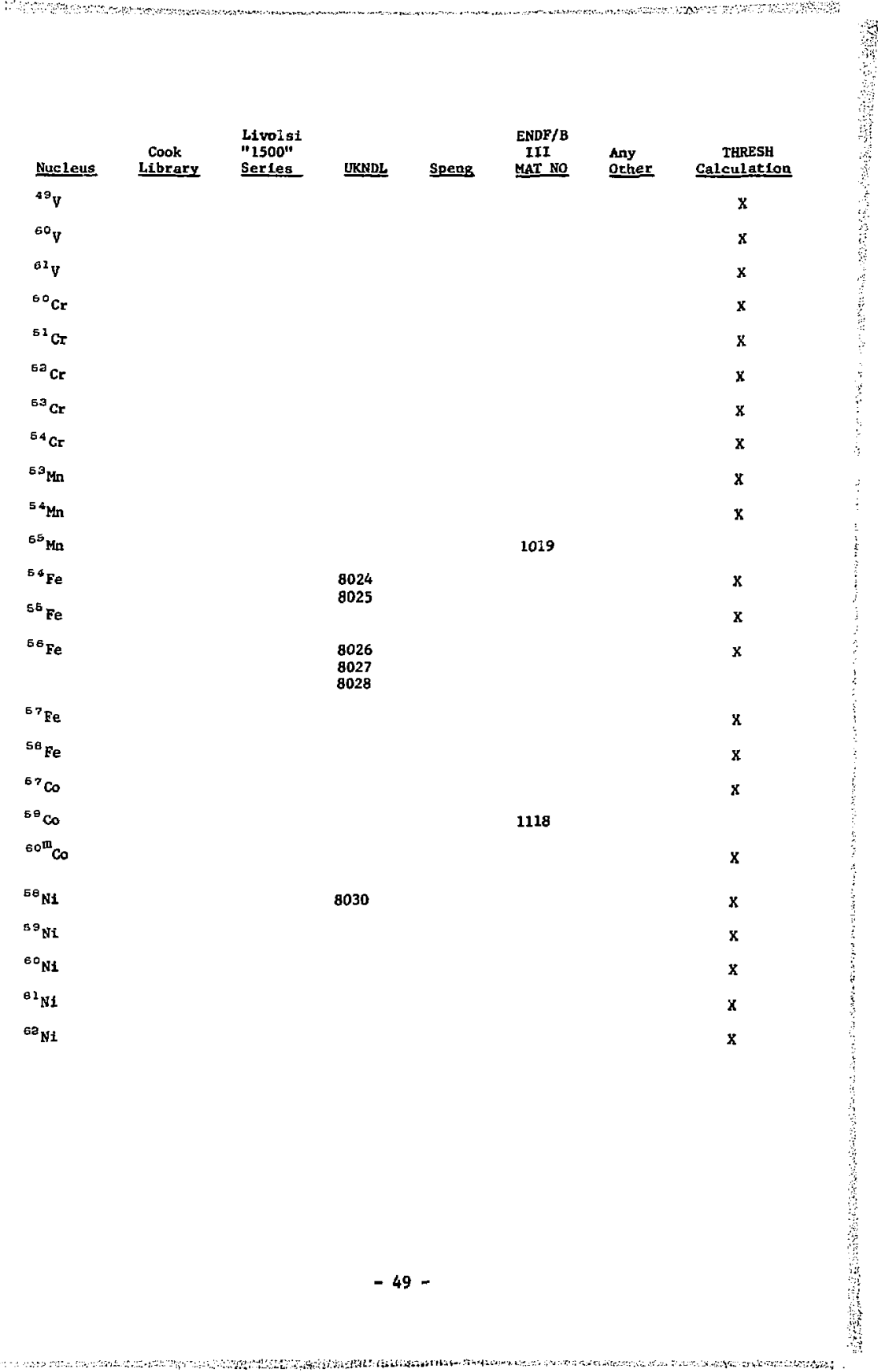




\begin{tabular}{|c|c|c|c|c|c|c|c|}
\hline Nucleus & $\begin{array}{c}\text { Chok } \\
\text { Llbraxy }\end{array}$ & $\begin{array}{l}\text { Livolal } \\
\text { "1500" } \\
\text { Serles }\end{array}$ & $\underline{\mathrm{UKNDL}}$ & Speng & $\begin{array}{l}\text { ENDF/B } \\
\text { III } \\
\text { MAT NO }\end{array}$ & $\begin{array}{l}\text { Any } \\
\text { Oeher }\end{array}$ & $\begin{array}{c}\text { ThiRESH } \\
\text { Calculation }\end{array}$ \\
\hline${ }^{63} \mathrm{~N} 1$ & & & & & & & $x$ \\
\hline${ }^{\mathrm{e}} \mathrm{NI}$ & & & & & & & $x$ \\
\hline${ }^{63} \mathrm{Cu}$ & & & & & 1085 & & \\
\hline${ }^{\mathrm{si}} \mathrm{Cu}$ & & & & & 1086 & & \\
\hline${ }^{6} \mathrm{Zn}$ & & & & & & & \\
\hline${ }^{65} \mathrm{Zn}$ & & & & & & & \\
\hline${ }^{84} \mathrm{Kr}$ & 7020 & 1521 & & & & & $\mathrm{x}$ \\
\hline${ }^{86} \mathrm{Kr}$ & 7021 & 1522 & & & & & $\mathrm{x}$ \\
\hline${ }^{8 \theta} \mathrm{Sr}$ & 7027 & & & & & & $\mathrm{x}$ \\
\hline${ }^{89} \mathrm{Sr}$ & 7028 & 1530 & & & & & $x$ \\
\hline${ }^{90} \mathrm{Sr}$ & 7029 & 1531 & & & & & $x$ \\
\hline${ }^{8 a} \mathbf{Y}$ & & & & & & & $\mathbf{x}$ \\
\hline${ }^{89} \mathbf{Y}$ & 7031 & & 8034 & & & & $x$ \\
\hline${ }^{9 \circ m_{Y}}$ & 7032 & & & & & & $x$ \\
\hline $91 \pi_{Y}$ & 7033 & & & & & & $\mathrm{x}$ \\
\hline${ }^{90} \mathrm{Zr}$ & 7035 & & 8035 & & & & $x$ \\
\hline${ }^{91} \mathrm{Zr}$ & 7036 & & & & & & $x$ \\
\hline${ }^{92} \mathrm{Zr}$ & 7037 & & & & & & $x$ \\
\hline${ }^{83} \mathrm{zr}$ & 7038 & & & & & & $x$ \\
\hline${ }^{94} \mathrm{Zr}$ & 7039 & & & & & & $x$ \\
\hline${ }^{85} \mathrm{zr}$ & 7040 & & & & 1202 & & $x$ \\
\hline${ }^{96} \mathrm{Zr}$ & 7041 & & & & & & $x$ \\
\hline${ }^{9 a} \mathbf{N b}$ & & & & & & & $x$ \\
\hline $9{ }^{\mathrm{Ml}} \mathrm{Nb}$ & & & & & & & $\mathbf{x}$ \\
\hline
\end{tabular}




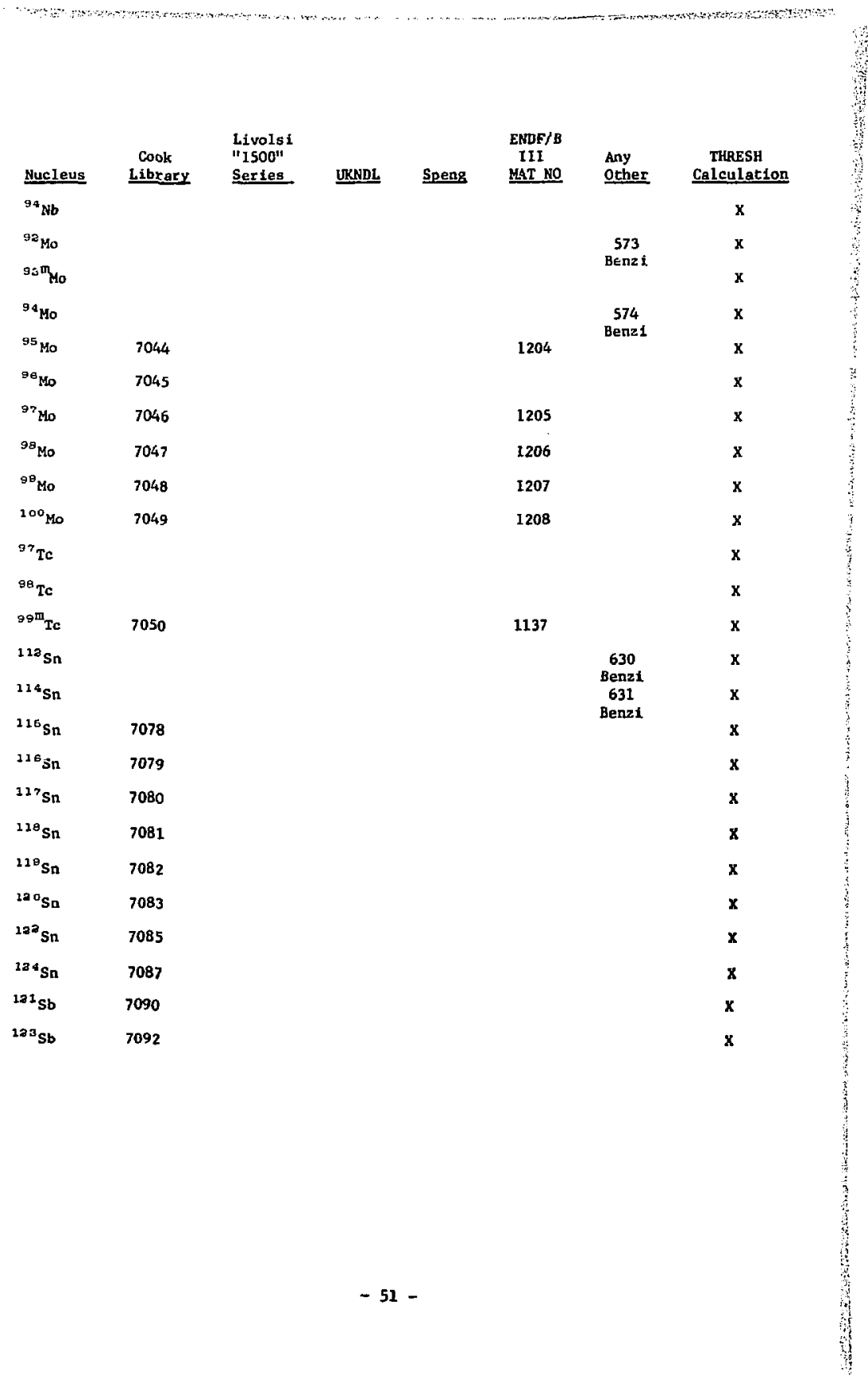




\begin{tabular}{|c|c|c|c|c|c|c|c|}
\hline Nucleus & $\begin{array}{c}\text { Cook } \\
\text { Library } \\
\end{array}$ & $\begin{array}{l}\text { LIvolsi } \\
\text { "1500" } \\
\text { Sertes }\end{array}$ & UKNDL & Speng & $\begin{array}{l}\text { ENDE/B } \\
\text { III } \\
\text { MAT NO }\end{array}$ & $\begin{array}{l}\text { Any } \\
\text { other }\end{array}$ & $\begin{array}{c}\text { THRESH } \\
\text { Calculation }\end{array}$ \\
\hline${ }^{129} I$ & 7100 & 1614 & & & & & $\mathrm{x}$ \\
\hline${ }^{134} \mathrm{Cs}$ & 7124 & 1628 & & & & & $x$ \\
\hline${ }^{236} \mathrm{Cs}$ & 7125 & 1629 & & & & & $x$ \\
\hline${ }^{136} \mathrm{Cs}$ & $\therefore 7126$ & 1630 & & & & & $x$ \\
\hline${ }^{137} \mathrm{Cs}$ & 7127 & 1631 & & & & & $x$ \\
\hline${ }^{134} \mathrm{Ba}$ & 7128 & 1633 & & & & & $x$ \\
\hline${ }^{195} \mathrm{Ba}$ & & 1634 & & & & & $\mathrm{x}$ \\
\hline${ }^{236} \mathrm{Ba}$ & 7129 & 1635 & & & & & $x$ \\
\hline${ }^{237} \mathrm{Ba}$ & 7130 & 1636 & & & & & $x$ \\
\hline${ }^{238} \mathrm{Ba}$ & 7131 & 1637 & & & & & $x$ \\
\hline${ }^{181} \mathrm{Ta}$ & & & & & 1126 & & $x$ \\
\hline${ }^{283} \mathrm{~W}$ & & & & & 1060 & & $x$ \\
\hline${ }^{203} \mathrm{~W}$ & & & & & 1061 & & $x$ \\
\hline $284 \mathrm{~W}$ & & & & & 1062 & & $x$ \\
\hline $18 e_{W}$ & & & & & 1063 & & $x$ \\
\hline${ }^{204} \mathrm{~Pb}$ & & & & & & & $x$ \\
\hline${ }^{306} \mathrm{~Pb}$ & & & & & & & $x$ \\
\hline $20{ }^{20} \mathrm{~Pb}$ & & & & & & & $x$ \\
\hline${ }^{08} \mathrm{~Pb}$ & & & & & & & $x$ \\
\hline $241 \mathrm{Am}$ & & & & & 1056 & & \\
\hline 242 & & & & & & & \\
\hline $243 \mathrm{Am}$ & & & & & 1057 & & \\
\hline
\end{tabular}

$-52-$ 


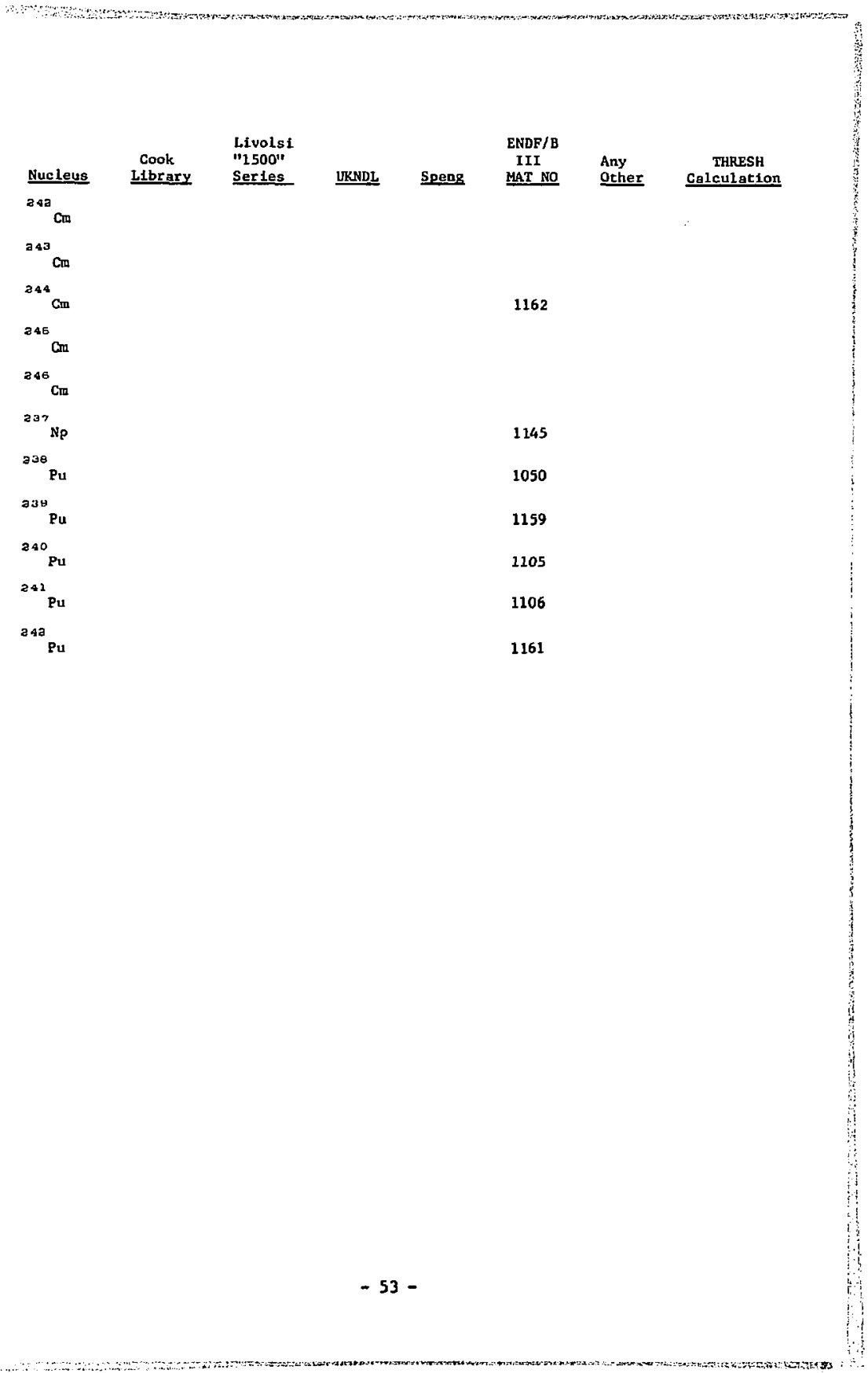




\section{References}

1. G. R. Hopkins (Ed). Proceedings of the First Topical Meeting on the Technology of Controlled Nucleat Fusion, San Diego, April 1974, CONF-740402-P2

2. H. C. Honeck, ENDF/B-Speciflcations for an Evaluated Nuclear Data for Reactor Applications BNL-50066 (ENDF-102) May 1966; Revised July 1967 and July 1968 by S. Pearlstein.

3. O. Ozer and D. Garber (Ed).ENDE/B Sumnary Dacumentation. BNL-17541 (ENDF-201) May 1973 and its up-dates. See also more detalled reports on individual evaluations.

4. K. Parker, The Aldermaston Nuclear Data Library as at May 1963. AWRE $0-70 / 63,1963$

5. R. J. Howerton, UCID-16376, Oct. 1973

6. M. K. Drake, BNL-17188 (ENDF-179) July 1972

7. C. Dev llers, J. Blachot, M. Lott, B. Nimal, N'Guyen Van Dat, J. P. Noe1, R deTourrel1, IAEA-SM-170/63, March 1973

8. Report $A E-R D-45$ (1972)

9. D. Woll, KFK-880 Dec. 1968

10. R. L. Simons and W. N. McElroy, BNWL-1312, May 1970

11. J. L. Cook, $A A E C / T M-549$, See also

E. K. Rose, AAEC/TM-587, March 1971

12. V. Benz 1 and G. Reffo. CCDN-NW/10 Dec. 1969

13. A. 2. Livolsi, BAW -409 , Nov. 1971

14. CCDN Newsletter; CCDN-NW/14, May 1972 
15. S. Pearlstein, Jour. Nucl. Energy 27, 81, 1973

16. A. H. Wapstra \& N. B. Gove, Nuclear Data Tables 9, 267 (1971)

17. S. Pearlstein, "Starter Evaluated Charged Particle Data Library" May 1974. Avallable on request.

18. S. Pearletein, Nuclear Data, 3A, 327, 1967, See also Nuc1. Sc1. and Eng. 23, 238, 1965

19. S. Blow, Jour. Nucl. Energy 26, 9, 1972

20. D. S. Macher, P. F. Bampton, R. E. Coles, G. James and P. J. Nind, AWRE 0 72/72, 1972

21. M. HHring, H. Vonach and E. J. Feicht, Z. Physik, 244, 352 (1971)

22. A. Paulgen and R. Widera, Z. Physik, 238, 23 (1970)

23. V. A. Konshin and M, N. Nikolaev, INDC(CCP) $-26 / U, 1972$

24. E. F. Plechaty and R. J. Howerton, UCID 16372 (1973) 